\title{
CODING GEOGRAPHIC AREAS ACROSS \\ CENSUS YEARS: CREATING CONSISTENT \\ DEFINITIONS OF METROPOLITAN AREAS
}

\author{
David A. Jaeger \\ Susanna Loeb \\ Sarah E. Turner \\ John Bound \\ Working Paper 6772 \\ http://www.nber.org/papers/w6772
NATIONAL BUREAU OF ECONOMIC RESEARCH
1050 Massachusetts Avenue
Cambridge, MA 02138 \\ October 1998
}

This coding effort benefited substantially from the resources of the Population Studies Center at the University of Michigan. In particular, the authors thank Lisa Neidert for her extraordinary efforts in helping us to use the Census materials and Lisa Lee for her research assistance. The views expressed here are those of the author and do not reflect those of the National Bureau of Economic Research.

(C) 1998 by David A. Jaeger, Susanna Loeb, Sarah E. Turner, and John Bound. All rights reserved. Short sections of text, not to exceed two paragraphs, may be quoted without explicit permission provided that full credit, including $\mathbb{C}$ notice, is given to the source. 
Coding Geographic Areas Across Census Years:

Creating Consistent Definitions of Metropolitan Areas

David A. Jaeger, Susanna Loeb,

Sarah E. Turner, and John Bound

NBER Working Paper No. 6772

October 1998

JEL No. J00

\begin{abstract}
This paper presents suggested matches for the geographical coding (geocoding) of metropolitan areas in the 1970,1980 , and 1990 Censuses. The Census Bureau used different definitions and taxonomies to describe the geography of metropolitan areas in these three Census years. As a result, the geographical areas referred to by the standard Census Bureau definitions differ among the three Census data sets. The geographic matching scheme explained in this paper attempts to maximize consistency over time for metropolitan areas in the U.S.
\end{abstract}

David A. Jaeger

Department of Economics

Hunter College

695 Park Avenue

New York, NY 10021-5085

David.Jaeger@hunter.cuny.edu

Susanna Loeb

Department of Economics

University of California -- Davis

Davis, CA 95616

sloeb@ucdavis.edu
Sarah E. Turner

School of Education and Dept. of Economics University of Virginia

405 Emmet Street

Charlottesville, VA 22903

sturner@virginia.edu

John Bound

Population Studies Center

University of Michigan

1225 South University Avenue

Ann Arbor, MI 48104

and NBER

jbound@umich.edu 


\section{Introduction}

This paper presents suggested matches for the geographical coding (geocoding) of metropolitan areas for use with the public use microsamples (PUMS) of the 1970, 1980, and 1990 Censuses. The Census Bureau used different definitions and taxonomies to describe the geography of metropolitan areas in these three Census years. As a result, the geographic areas referred to by the standard Census Bureau definitions differ among the three Census data sets. The geographic matching scheme explained in this paper attempts to maximize consistency over time for metropolitan areas in the U.S.

These geocodings first appeared in two projects that used metropolitan areas as proxies for well-defined, independent labor markets. Building on the earlier work of Loeb described below, Jaeger (1995) constructed geographically consistent definitions of the 50 largest metropolitan areas between the 1980 and 1990 Censuses to examine the substitutability of immigrants and natives in different skill categories. He also examined the impact of immigration on the wages of native workers in those 50 metropolitan areas. Bound and Holzer (1996) examined the effect of labor demand shifts and population adjustments on economic outcomes of specific demographic groups during the 1980s. For this analysis, Loeb and Turner extended Jaeger's geocodings to cover the 132 metropolitan areas with 1990 populations in excess of 250,000. Prior to these studies, Loeb generated a 1970-80-90 match for an initial inquiry on local labor markets by Bound and Holzer. ${ }^{1}$

\footnotetext{
${ }^{1}$ Loeb's work built on an earlier 1970-80 match of 52 metropolitan areas done by Marshall Cummings for work done by Bound and Holzer (1993) using the 1970 and 1980 Censuses.
} 
The geographic coding available in the 1970 Census necessitates more limited, and less inclusive, definitions of the metropolitan areas. This results in a less-precise mapping than is possible between 1980 and 1990 alone. In this sense, the geographic coding of the PUMS data for the three Census years implies that the 1970/1980/1990 matching is cruder than the 1980/1990 matching. Since there are certainly many additional questions that might be addressed using these data, the purpose of this note is to provide a guide to how the metropolitan area mappings were made from which others may benefit.

Section II of the paper briefly summarizes the various terms and resources important in using data with metropolitan areas as the focal geographic unit. Section III provides a step-by-step explanation of the matching process between 1980 and 1990, with a summary of the imperfections in this process. Section IV discusses the matching of 1970, 1980 and 1990 metropolitan areas and explains why the changes in coding during that time inhibit the process of matching without the introduction of substantial mismatches across years.

\section{Census Micro Data and the Classification of Metropolitan Areas}

In 1990, the Census Bureau defines a Metropolitan Area (MA) as:

a large population nucleus, together with adjacent communities that have a high degree of economic and social integration with that nucleus...Each MA must contain either a place with a minimum population of 50,000 or a Census Bureau-defined urbanized area and a total MA population of at least 100,000 (75,000 in New England). An MA comprises one or more central counties (cities and towns in New England) that have close economic and social relationships with the central county. An outlying county must have a 
specified level of commuting to the central counties and must meet certain standards regarding metropolitan character, such as population density, urban population, and population growth.

Using this concept as a starting point, our objective was to construct metropolitan areas that maximize geographic consistency across Census years. While this is a relatively straightforward task in principle, changes in federal definitions of metropolitan areas, as well as a change in the coding scheme to designate sub-areas comprising these metropolitan areas, complicate this process considerably.

One source of confusion and complication in many analyses discussing metropolitan areas is the distinction among a veritable "alphabet soup" of metropolitan designations. Various "types" of MAs, which are not necessarily mutually exclusive, and the Census years in which the terms applied include:

- MSA (90): relatively freestanding and not closely associated with other MAs, typically surrounded by non-metropolitan areas; the title of an MSA contains the name of its largest city and up to two additional city names.

- CMSA (90): a consolidated metropolitan area is an MA of more than 1 million people which may included one or more PMSAs (see below); this concept provides an umbrella classification for cities whose economies are closely tied. ${ }^{2}$

- PMSA (90): a primary metropolitan statistical area defines a large urbanized county or cluster of counties that demonstrate very strong internal economic and social links within a CMSA

- SMSA (80): An SMSA consists of one or more entire counties or county equivalents (in New England, towns and cities are the basic units).

\footnotetext{
${ }^{2}$ For example, in 1980 New York City, Jersey City, and Newark were considered to be separate metropolitan areas. In 1990, all three -- along with a substantial portion of New Jersey, and several counties in New York State and Connecticut -- are considered to be part of the New York--New Jersey--Long Island CMSA.
} 
Although counties are the primary political units in most states, other designations are used in states like Louisiana where parishes are the primary geographical division and in New England areas where cities and towns are often more meaningful designations.

MA designations are managed by the Federal Office of Management and Budget, following uniform standards. Unfortunately, since the SMSA to PMSA/CMSA/MSA mappings are not one-to-one, it is not possible to simply map from one scheme to the other; rather, it is necessary to examine the component parts of these metropolitan classifications to put the pieces together as consistently as possible.

For the 1990 Census microdata, the building blocks in creating metropolitan areas are the Public Use Microdata Areas (or PUMAs). PUMAs comprise population groups of at least 100,000 persons and are designated by five-digit numbers that are unique within states. While the Census Bureau records data at the more disaggregate levels of the block, the block area and the tract, the PUMA is the most disaggregate unit of analysis available to the researcher with the microdata records. ${ }^{3}$ Depending on the population density of the specific area and the geographic range of the respective counties, PUMAs may define a subset (several Census tracts) of a single county (e.g., Westchester County in New York State comprises PUMAs 04401 through 04405 plus 04500 ) or, in less densely populated areas, the PUMA might consist of several counties, all with quite small populations (e.g., PUMA 03500 in New York State comprises Cortland, Tioga, and Tompkins Counties).

\footnotetext{
${ }^{3}$ The Census Bureau does release aggregate or average data at more disaggregate units such as the tract.
} 
For the 1980 Census microdata, the county group is the primary building block used in constructing the metropolitan area. While the county group serves the same functional purpose as the PUMA, the definitions are not entirely coincident with PUMAs. In general, county groups tend to comprise somewhat larger geographic areas than PUMAs, though this is not universally true. Thus, our objective is to "add up" county groups in 1980 and PUMAs in 1990 to produce consistent geographical definitions for each metropolitan area.

\section{The Mechanics of the 1990 to 1980 match}

The primary tools for matching 1980 and 1990 data are the geographical equivalency files for each year. ${ }^{4}$ For matching 1990 and 1980 data, we focus on CMSA definitions where applicable, because CMSAs are likely to be better approximations of local labor markets than PMSAs. In total, we matched county groups and PUMAs for 132 MSAs, representing all metropolitan areas with populations larger than 250,000.

The steps to the matching process include:

1. Match PUMAs to MSAs/CMSAs in the 1990 Census using the Geographical Equivalency file.

\footnotetext{
${ }^{4}$ The geographic equivalency file for the 5 percent PUMS can be found at ftp://ftp.psc.lsa.umich.edu/pub/census/pums/1980/5pct/equiv/ and
} 
As a first step, we are able to eliminate those PUMAs composed of entirely nonmetropolitan areas. Next, when PUMAs reflect a geographical area entirely within a CMSA/MSA the PUMA is allocated to the MSA. Cases in which the PUMA is split between (possibly multiple) metropolitan areas and/or non-metropolitan areas present more difficulty. In cases where the PUMA encompasses multiple metropolitan areas, it is assigned to a single metropolitan area based on geography and the relative populations of the different metropolitan areas within the PUMA. In other words, the PUMA is assigned to the MSA with a greatest share of the PUMA's population. For PUMAs including both a metropolitan and non-metropolitan areas, we assigned the entire PUMA to the metropolitan area. Thus, our definition of CMSAs and MSAs is slightly different from those defined by the Bureau of the Census and includes some non-metropolitan areas.

\section{Match 1980 County Groups to 1990 MSA/CMSA Definitions.}

Having redefined the CMSAs/MSAs based on information available in the 1990 PUMS file, we then match the 1980 county groups to those definitions. In most cases, we were able to match the geographies exactly or nearly exactly.

Table 1, which gives extracts from the 1980 and 1990 geographic equivalency files for the area around Little Rock, Arkansas, helps to illustrate this process more clearly. Looking at the 1990 equivalency extract, we see that Little Rock (CMSA 4400) appears in PUMA 1500 and PUMA 1600. All of PUMA 1500 is in Little Rock so this is immediately

ftp://ftp.psc.lsa.umich.edu/pub/census/pums/1990/5pct/equiv/, for 1980 and 1990, 
included in our definition of Little Rock. Only part of PUMA 1600 is in the CMSA definition of Little Rock. However, since the remainder of PUMA 1600 is not in any other metropolitan area, this PUMA is also included in our definition. We see from the extract that, while the Census definition included only Pulaski (119), Saline (125), Faulkner (045), and Lonoke (085) counties. Ours includes Monroe (095) and Prairie (117) counties as well.

Next look at the 1980 geographic equivalency file extract. We would like to select the county groups so as to match the counties in Little Rock between 1980 and 1990 . County group 007 includes counties 045, 085, 095, and 117, all of which are in our definition of Little Rock. We therefore include county group 007 in our 1980 sample. Similarly county groups 008 and 009 include parts of county 119 so they are included as well. Finally, county group 010 has both county 125 and the balance of Pulaski County and so also is included in our 1980 definition. In this case we have a perfect match between the two years.

Table 2 presents the results of this matching process for the 132 MSAs with 1990 populations greater than 250,000 . The first column gives the name of the metropolitan area and the second, the state number. Columns two and three give the 1980 county group numbers and the 1990 PUMA numbers that comprise our definition of the MA.

Table 3 describes each C/MSA match by its 1990 population based on the geographic areas included in our 1990 definition, the ratio of our definition to the Census Bureau definition (as a measure of the similarity of the Census Bureau definition and our respectively. 
definition), the ratio of the 1990 population based on our definition to the 1990 population based on the county group definition (as a measure of how well the 1980 and 1990 definitions match), and the percent of the C/MSA that is considered a metropolitan area in our 1990 definition. The matches are generally quite close, if not exact. Our definition contains somewhat larger populations than the Census Bureau's for some metropolitan areas.

SAS code which implements these matches is available at http://www.nber.org/census-geocodes.

\section{Matching 1970, 1980, and 1990}

The process for matching 1970, 1980 and 1990 metropolitan areas was similar to the one described above for the later two years. However, the geographic (county group) codes available in the 1970 Census allow for far less precision than in the later years. In most cases, the metropolitan area, as defined in 1970, is composed of one county group. Trying to expand this definition to include surrounding areas often entails including most of the remainder of the state. Moreover, the 1970 definitions of the metropolitan areas tend to be substantially smaller than in 1980 or 1990 . The choice then becomes between too small a definition, which may leave out a substantial part of the area labor market, or too large a definition, which may defeat the purpose of using metropolitan areas instead of states as the unit of analysis. The situation is not so dark for all areas. 
Table 4 presents the 1970-80-90 matches for 125 metropolitan areas that are identifiable in the 1970 PUMS data. The third column of this table gives the names of the counties included in the 1970 definition of each metropolitan area. The fourth column gives the names of other counties that were included in either our 1980 or 1990 definition. It is important to note that the 1980 and 1990 definitions presented here are not the same as those for the 1980-90 match since they are based closely on the 1970 Census definition, not the 1990 Census definition. Columns five and six give the county numbers and 1980 county populations. The three remaining columns give the 1970 county group numbers, the 1980 county group numbers, and the 1990 PUMA numbers to be included in the match. In most cases all these numbers are in bold text and should be included in the new definitions. In a few cases, plain text numbers are included. We do not include these in our match. However, the matches including and not including these plain text county groups or PUMAs were close enough that we provide them in the table so that the reader may decide his/her own preference for including or excluding these areas. The final line in the table for each metropolitan area describes the precision of the fit.

To see how this matching was accomplished, again consider Little Rock, Arkansas. In 1970, this metropolitan area was defined as Saline and Pulaski Counties alone. For the 1980 these counties correspond precisely to county groups 008, 009 and 010, while in 1990 they are both included in PUMA 1500. As is the case for the 1980-90 mapping, this is a perfect match. However, it also is a substantially more limited definition of Little Rock. As a second example, consider Miami, Florida. The 1970 definition includes only Dade County. In 1990 we can match Dade County exactly with PUMAs 03601, 03602, 03700, 
03800, and 03901-03909. In 1980, on the other hand, in order to get all of Dade County we have to use county group 053, which includes Monroe County as well. Based on the 1980 population figures, we then have a perfect match between 1970 and 1990 but have 3.89\% more people in our 1980 definition. From the plain text PUMAs we can also see that in order to match 1980 and 1990 more precisely we would have to include PUMA 02400 which would give us Collier County, as well, taking us further from the 1970 definition. We chose not to do this for our match. Overall, 63 of the 125 metropolitan areas are perfectly matched across the three years. The precision of the other matches varies considerably. The table indicates for which areas the match is not precise; those areas without notes match exactly.

\section{Conclusion}

Matching the geographies of metropolitan areas is complicated by a lack of consistent definitions in the underlying data. Using the constituent geographic units county groups in 1970 and 1980, and PUMAs in 1990, we propose two matches for use with PUMS data from the 1970, 1980, and 1990 Censuses. In the first, we provide a match between the 1980 and 1990 geographies, while in the second, we provide a less-precise match between the geographies in all three Census years. In this match we have attempted to maximize the geographic consistency of our definitions across years to facilitate the analysis of economic and social outcomes with the Census. 


\section{References}

Bound, John and Harry Holzer (1993) "Industrial Shifts, Skill Levels, and the Labor Market for White and Black Males," Review of Economics and Statistics, 75 (August), 387-396.

Bound, John and Harry Holzer (1996) "Demand Shifts, Population Adjustments, and Labor Market Outcomes During the 1980s," NBER Working Paper 5685, July.

Census of Population and Housing (1992) “1990: Public Use Microdata Sample U.S. Technical Documentation" prepared by the Bureau of the Census, Washington, D.C.

Census of Population and Housing (1983) “1980: Public Use Microdata Sample U.S. Technical Documentation" prepared by the Bureau of the Census, Washington, D.C.

Jaeger, David A. (1995) Essays in Empirical Labor Economics, Ph.D. Dissertation, University of Michigan Department of Economics. 
Table 1: Extracts from Geographical Equivalency File, 1980 and 1990, Little Rock, AR

\section{Equivalency File}

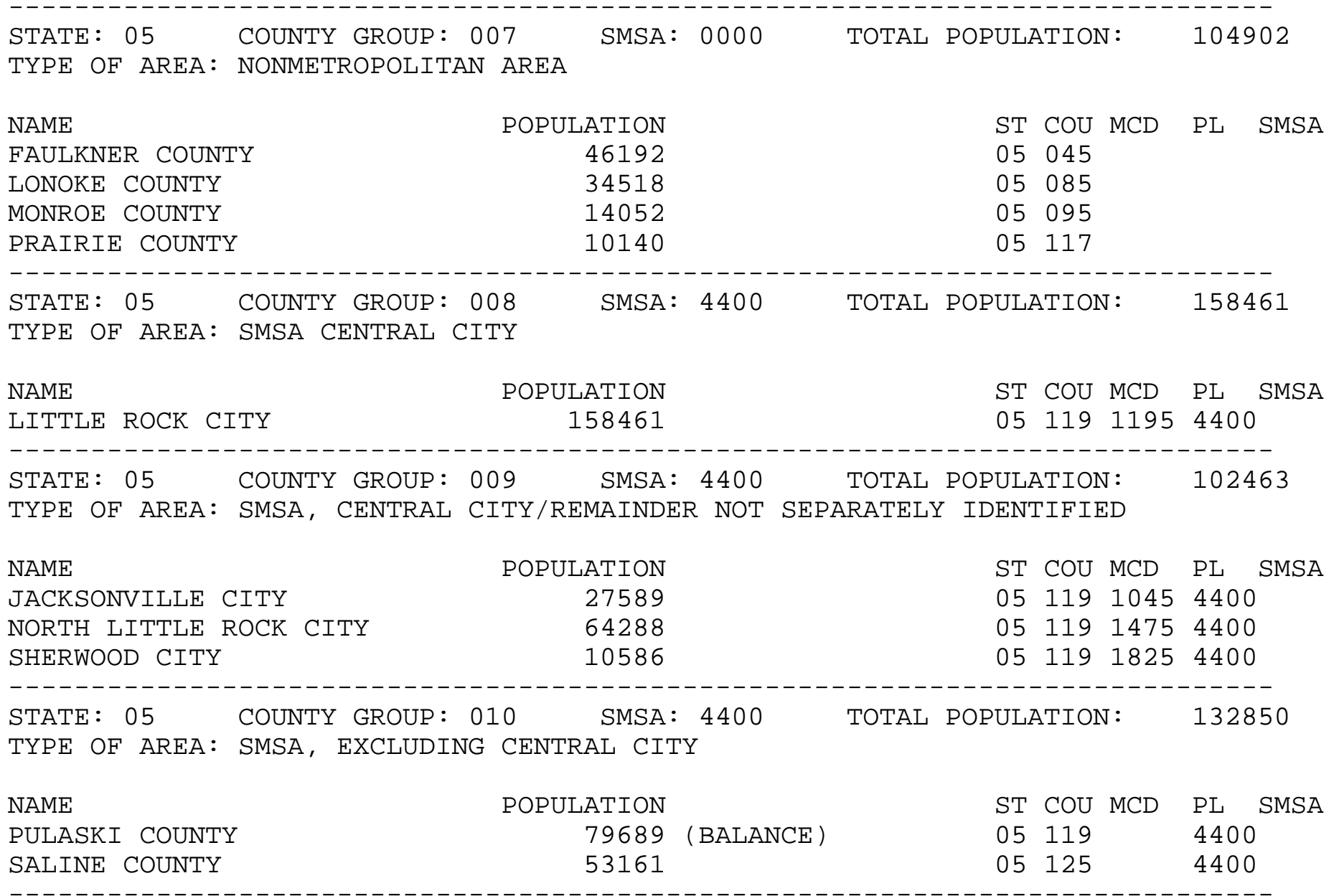




\section{Table 1, continued}

\section{Equivalency File}

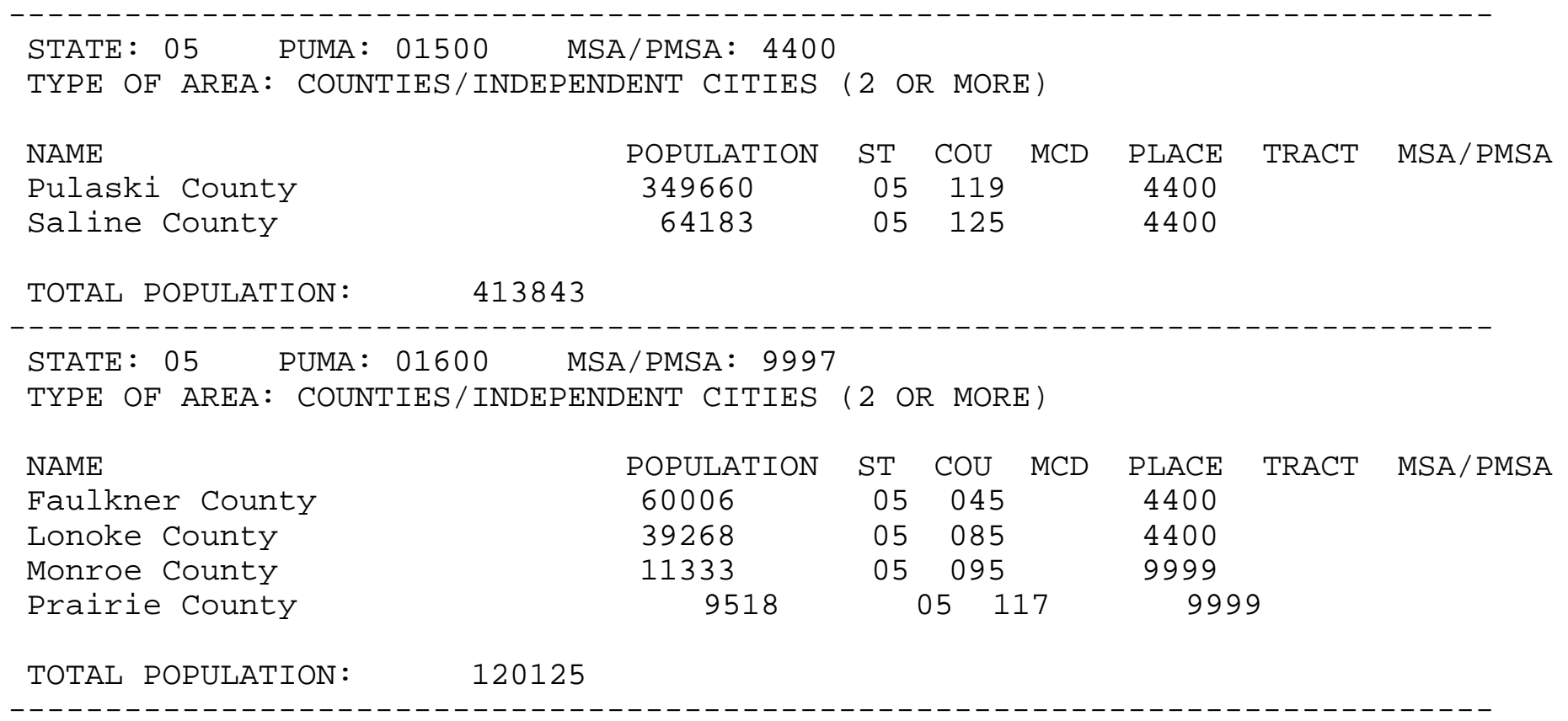


Table 2: 1980 -1990 Matches Based on 1990 MSA/CMSA Definitions

\begin{tabular}{|c|c|c|c|c|}
\hline MSA & C/MSA Name & $\begin{array}{l}\text { State } \\
\text { Num. }\end{array}$ & $\begin{array}{c}1980 \\
\text { County } \\
\text { Group }\end{array}$ & $\begin{array}{c}1990 \\
\text { PUMA }\end{array}$ \\
\hline \multirow[t]{2}{*}{0160} & Albany-Schenectady-Troy, NY & 36 & $5-9$ & $800-1200$ \\
\hline & & 36 & 33 & 3800 \\
\hline 0200 & Albuquerque, NM & 35 & $4-5$ & 201-204 \\
\hline 0240 & Allentown-Bethlehem, PA-NJ & 42 & $51-54$ & 2001-2102 \\
\hline 0460 & Appleton-Oshkosh-Neenah, WI & 55 & $5-6$ & $400-500$ \\
\hline \multirow[t]{4}{*}{0520} & Atlanta, GA & 13 & $4-10$ & 800 \\
\hline & & 13 & 12 & $1500-2103$ \\
\hline & & 13 & 27 & 2300 \\
\hline & & 13 & & 3000 \\
\hline 0560 & Atlantic City, NJ & 34 & 38 & $100-200$ \\
\hline \multirow[t]{3}{*}{0600} & Augusta, GA-SC & 13 & $25-26$ & 500 \\
\hline & & 13 & & 2200 \\
\hline & & 45 & 16 & 1800 \\
\hline \multirow[t]{2}{*}{0640} & Austin, TX & 48 & $44-45$ & $4901-5200$ \\
\hline & & 48 & 66 & \\
\hline 0680 & Bakersfield, CA & 6 & $36-37$ & $4900-5002$ \\
\hline \multirow[t]{4}{*}{0720} & Baltimore, MD & 24 & $4-8$ & $201-306$ \\
\hline & & 24 & 12 & $500-600$ \\
\hline & & 24 & & $1000-1100$ \\
\hline & & 24 & & $1501-1504$ \\
\hline 0760 & Baton Rouge, LA & 22 & $14-17$ & $1200-1500$ \\
\hline 0840 & Beaumont-Port Arthur, TX & 48 & $54-56$ & $5900-6100$ \\
\hline \multirow[t]{2}{*}{0960} & Binghampton, NY & 36 & 17 & $3500-3602$ \\
\hline & & 36 & $29-30$ & \\
\hline 1000 & Birmingham, AL & 1 & $9-11$ & $1700-1806$ \\
\hline \multirow[t]{3}{*}{1123} & Boston-Lawernce-Salem-Lowell-Brockton, MA & 25 & $11-33$ & $1400-3800$ \\
\hline & & 33 & 3 & 501 \\
\hline & & 33 & & 503 \\
\hline 1240 & Brownsville-Harlingen, TX & 48 & 38 & $4201-4202$ \\
\hline
\end{tabular}


Table 2, continued

\begin{tabular}{|c|c|c|c|c|}
\hline MSA & C/MSA Name & $\begin{array}{l}\text { State } \\
\text { Num. }\end{array}$ & $\begin{array}{c}1980 \\
\text { County } \\
\text { Group } \\
\end{array}$ & $\begin{array}{c}1990 \\
\text { PUMA }\end{array}$ \\
\hline \multirow[t]{2}{*}{1282} & Buffalo-Niagara Falls, NY (C) & 36 & $23-25$ & $2301-2302$ \\
\hline & & 36 & & 2500-3003 \\
\hline 1320 & Canton, $\mathrm{OH}$ & 39 & $27-28$ & $5001-5003$ \\
\hline 1440 & Charleston, SC & 45 & $19-21$ & $1100-1202$ \\
\hline 1480 & Charleston, WV & 54 & $8-9$ & 700 \\
\hline \multirow[t]{2}{*}{1520} & Charlotte-Gastonia-Rock Hill NC-SC & 37 & $36-40$ & $801-1200$ \\
\hline & & 45 & 6 & 500 \\
\hline \multirow[t]{2}{*}{1560} & Chattanooga, TN-GA & 13 & 1 & 300 \\
\hline & & 47 & $15-17$ & $1000-1300$ \\
\hline \multirow[t]{5}{*}{1602} & Chicago-Gary-Lake County, IL-IN-WI (C) & 17 & $32-38$ & $3001-3502$ \\
\hline & & 18 & $1-4$ & $800-1000$ \\
\hline & & 18 & & 1200 \\
\hline & & 18 & & $3700-3900$ \\
\hline & & 55 & 21 & 2000 \\
\hline \multirow[t]{5}{*}{1642} & Cincinnati-Hamilton, OH-KY-IN (C) & 18 & 36 & 400 \\
\hline & & 21 & $1-2$ & $1300-1400$ \\
\hline & & 39 & $51-55$ & 3000 \\
\hline & & 39 & & 3300 \\
\hline & & 39 & & $5301-5406$ \\
\hline \multirow[t]{3}{*}{1692} & Cleveland-Akron-Lorain, $\mathrm{OH}(\mathrm{C})$ & 39 & $6-18$ & 400 \\
\hline & & 39 & $23-25$ & $800-900$ \\
\hline & & 39 & & $3700-4704$ \\
\hline 1720 & Colorado Springs, CO & 8 & 14 & $1000-1100$ \\
\hline 1760 & Columbia, SC & 45 & $13-15$ & $1601-1700$ \\
\hline \multirow[t]{4}{*}{1840} & Columbus, $\mathrm{OH}$ & 39 & $41-44$ & 1700 \\
\hline & & 39 & 56 & 2300 \\
\hline & & 39 & & $2700-2800$ \\
\hline & & 39 & & $5101-5107$ \\
\hline \multirow[t]{3}{*}{1880} & Corpus Christi, TX & 48 & 35 & 4000 \\
\hline & & 48 & 39 & $4301-4302$ \\
\hline & & 48 & & 4800 \\
\hline
\end{tabular}


Table 2, continued

\begin{tabular}{|c|c|c|c|c|}
\hline MSA & C/MSA Name & $\begin{array}{l}\text { State } \\
\text { Num. }\end{array}$ & $\begin{array}{c}1980 \\
\text { County } \\
\text { Group } \\
\end{array}$ & $\begin{array}{c}1990 \\
\text { PUMA }\end{array}$ \\
\hline \multirow[t]{2}{*}{1922} & Dallas-Fort Worth, TX (C) & 48 & $13-25$ & 1500 \\
\hline & & 48 & & 1800-2904 \\
\hline \multirow[t]{3}{*}{1960} & Davenport-Rock Island-Moline, IA-IL & 17 & 7 & 600 \\
\hline & & 17 & $10-11$ & 1000 \\
\hline & & 19 & 16 & 1400 \\
\hline \multirow[t]{3}{*}{2000} & Dayton-Springfield, $\mathrm{OH}$ & 39 & $45-50$ & $2100-2200$ \\
\hline & & 39 & & 2600 \\
\hline & & 39 & & $5201-5205$ \\
\hline 2020 & Daytona Beach, FL & 12 & $14-15$ & 900 \\
\hline 2082 & Denver-Boulder, CO (C) & 8 & $6-12$ & $101-702$ \\
\hline 2120 & Des Moines, IA & 19 & $8-11$ & $700-1000$ \\
\hline \multirow[t]{2}{*}{2162} & Detroit-Ann Arbor, MI (C) & 26 & 24 & 2200 \\
\hline & & 26 & $33-59$ & $3000-4400$ \\
\hline 2320 & El Paso, TX & 48 & 32 & $3701-3705$ \\
\hline 2360 & Erie, PA & 42 & $1-2$ & $101-102$ \\
\hline 2400 & Eugene-Springfield, OR & 41 & $9-10$ & $700-800$ \\
\hline \multirow[t]{2}{*}{2440} & Evansville, IN-KY & 18 & $31-33$ & 500 \\
\hline & & 18 & & 2400 \\
\hline 2560 & Fayetteville, NC & 37 & $32-33$ & $3000-3100$ \\
\hline 2640 & Flint, MI & 26 & $21-23$ & $2000-2102$ \\
\hline 2700 & Fort Myers-Cape Coral FL & 12 & 31 & $3001-3003$ \\
\hline 2710 & Fort Pierce, FL & 12 & 30 & $2700-2800$ \\
\hline \multirow[t]{2}{*}{2760} & Fort Wayne, IN & 18 & $9-12$ & $1700-1800$ \\
\hline & & 18 & & $2600-2700$ \\
\hline 2840 & Fresno, CA & 6 & $27-28$ & $4000-4100$ \\
\hline \multirow[t]{2}{*}{3000} & Grand Rapids MI & 26 & $13-15$ & $1300-1700$ \\
\hline & & 26 & 17 & \\
\hline \multirow[t]{2}{*}{3120} & Greensboro-Winston-Salem-High Point, NC & 37 & $7-13$ & $1300-1700$ \\
\hline & & 37 & & $2000-2200$ \\
\hline 3160 & Greenville-Spartanburg, SC & 45 & $2-5$ & $100-302$ \\
\hline \multirow[t]{2}{*}{3240} & Harrisburg-Lebanon-Carlisle, PA & 42 & $41-45$ & 2200 \\
\hline & & 42 & & $3601-3700$ \\
\hline
\end{tabular}


Table 2, continued

\begin{tabular}{|c|c|c|c|c|}
\hline MSA & C/MSA Name & $\begin{array}{l}\text { State } \\
\text { Num. }\end{array}$ & $\begin{array}{c}1980 \\
\text { County } \\
\text { Group } \\
\end{array}$ & $\begin{array}{c}1990 \\
\text { PUMA }\end{array}$ \\
\hline \multirow[t]{2}{*}{3283} & Hartford-New Britain-Middletown-Bristol CT & 9 & 11 & $200-1000$ \\
\hline & & 9 & $14-21$ & 2500 \\
\hline 3320 & Honolulu, HI & 15 & $1-2$ & 301-307 \\
\hline \multirow[t]{3}{*}{3362} & Houston-Galveston-Brazoria, TX (C) & 48 & $50-51$ & $5500-5600$ \\
\hline & & 48 & $57-64$ & $6200-6908$ \\
\hline & & 48 & & 7200 \\
\hline \multirow[t]{3}{*}{3400} & Huntington-Ashland, WV-KY-OH & 21 & 11 & 1100 \\
\hline & & 39 & 59 & 3500 \\
\hline & & 54 & $10-11$ & 800 \\
\hline \multirow[t]{2}{*}{3480} & Indianapolis, IN & 18 & $21-24$ & $101-107$ \\
\hline & & 18 & & $3300-3500$ \\
\hline 3560 & Jackson, MS & 28 & $8-10$ & $800-1000$ \\
\hline \multirow[t]{2}{*}{3600} & Jacksonville, FL & 12 & $7-9$ & 200 \\
\hline & & 12 & & $1000-1100$ \\
\hline \multirow[t]{3}{*}{3660} & Johnson City-Kingsport-Bristol, TN-VA & 47 & $25-27$ & $100-300$ \\
\hline & & 51 & $9-10$ & 200 \\
\hline & & 51 & & 3200 \\
\hline \multirow[t]{2}{*}{3760} & Kansas City MO-KS & 20 & $9-11$ & $901-1100$ \\
\hline & & 29 & $7-12$ & $700-1005$ \\
\hline 3810 & Killeen-Temple, TX & 48 & $46-47$ & $5300-5400$ \\
\hline 3840 & Knoxville, TN & 47 & $21-24$ & $600-900$ \\
\hline 3980 & Lakeland-Winter Haven FL & 12 & 20 & 4600 \\
\hline 4000 & Lancaster, PA & 42 & $46-48$ & $3501-3503$ \\
\hline \multirow[t]{2}{*}{4040} & Lansing-East Lansing MI & 26 & 18 & $1700-1900$ \\
\hline & & 26 & 20 & \\
\hline 4120 & Las Vegas NV & 32 & $1-2$ & 201-205 \\
\hline 4280 & Lexington-Fayette, $\mathrm{KY}$ & 21 & $6-8$ & $1600-1802$ \\
\hline 4400 & Little Rock-North Little Rock, AR & 5 & $7-10$ & $1500-1600$ \\
\hline \multirow[t]{3}{*}{4472} & Los Angeles-Anaheim-Riverside, CA (C) & 6 & $38-50$ & 3700 \\
\hline & & 6 & & $4200-4808$ \\
\hline & & 6 & & $5200-7207$ \\
\hline \multirow[t]{2}{*}{4520} & Louisville, KY-IN & 18 & $34-35$ & $200-300$ \\
\hline & & 21 & $3-5$ & $1900-2103$ \\
\hline \multirow[t]{2}{*}{4680} & Macon-Warner Robins, GA & 13 & $13-14$ & 400 \\
\hline & & 13 & & 3100 \\
\hline
\end{tabular}


Table 2, continued

\begin{tabular}{|c|c|c|c|c|}
\hline MSA & C/MSA Name & $\begin{array}{l}\text { State } \\
\text { Num. }\end{array}$ & $\begin{array}{c}1980 \\
\text { County } \\
\text { Group } \\
\end{array}$ & $\begin{array}{c}1990 \\
\text { PUMA } \\
\end{array}$ \\
\hline 4720 & Madison, WI & 55 & $17-18$ & $1600-1700$ \\
\hline 4880 & McAllen-Edinburg-Mission, TX & 48 & $36-37$ & 4101-4103 \\
\hline 4900 & Melbourne-Titusville-Palm Bay, FL & 12 & 16 & $1200-1400$ \\
\hline \multirow[t]{3}{*}{4920} & Memphis, TN-AR-MS & 5 & 5 & 600 \\
\hline & & 28 & 1 & 100 \\
\hline & & 47 & $1-3$ & $1800-2000$ \\
\hline 4992 & Miami-Fort Lauderdale, FL (C) & 12 & $36-53$ & 3200-3909 \\
\hline 5082 & Milwaukee-Racine, WI (C) & 55 & $22-26$ & $2100-2400$ \\
\hline \multirow[t]{3}{*}{5122} & Minneapolis-St.Cloud MN-WI (C) & 27 & $14-25$ & 900 \\
\hline & & 27 & & $1100-2400$ \\
\hline & & 55 & 12 & 1100 \\
\hline 5160 & Mobile, AL & 1 & $19-21$ & $700-800$ \\
\hline 5170 & Modesto, CA & 6 & $22-23$ & $2400-2500$ \\
\hline \multirow[t]{2}{*}{5240} & Montgomery, AL & 1 & 17 & 1300 \\
\hline & & 1 & & 1500 \\
\hline \multirow[t]{2}{*}{5360} & Nashville, TN & 47 & $8-11$ & $501-505$ \\
\hline & & 47 & & $2300-2500$ \\
\hline \multirow[t]{2}{*}{5480} & New Haven-Meriden CT & 9 & $7-10$ & $2000-2200$ \\
\hline & & 9 & & 2400 \\
\hline 5520 & New London-Norwich, CT-RI & 9 & $12-13$ & $2600-2700$ \\
\hline \multirow[t]{2}{*}{5560} & New Orleans LA & 22 & 19 & 1700 \\
\hline & & 22 & $21-26$ & $1901-2300$ \\
\hline \multirow[t]{6}{*}{5602} & N.Y.-North. N.J.-Long Island, NY-NJ-CT (C) & 9 & $1-6$ & $1100-1700$ \\
\hline & & 9 & 24 & 2300 \\
\hline & & 34 & $1-37$ & $400-4400$ \\
\hline & & 34 & $51-52$ & \\
\hline & & 36 & $34-44$ & $2401-2412$ \\
\hline & & 36 & & $4000-5414$ \\
\hline \multirow[t]{3}{*}{5720} & Norfolk-Virginia Beach-Newport News VA & 51 & $16-21$ & 2300 \\
\hline & & 51 & 26 & $2500-3100$ \\
\hline & & 51 & & 3300 \\
\hline 5880 & Oklahoma City, OK & 40 & $10-13$ & $900-1100$ \\
\hline
\end{tabular}


Table 2, continued

\begin{tabular}{|c|c|c|c|c|}
\hline MSA & C/MSA Name & $\begin{array}{l}\text { State } \\
\text { Num. }\end{array}$ & $\begin{array}{c}1980 \\
\text { County } \\
\text { Group } \\
\end{array}$ & $\begin{array}{c}1990 \\
\text { PUMA } \\
\end{array}$ \\
\hline \multirow[t]{2}{*}{5920} & Omaha, NE-IA & 19 & 19 & 1700 \\
\hline & & 31 & 6-7 & 900-1004 \\
\hline 5960 & Orlando, FL & 12 & $17-19$ & $1600-2300$ \\
\hline 6080 & Pensacola, FL & 12 & 1 & 100 \\
\hline 6120 & Peoria, IL & 17 & $8-9$ & 700-900 \\
\hline \multirow[t]{4}{*}{6162} & Philadelphia-Wilmington-Trenton, PA-NJ-DE-MD (C) & 10 & 1 & 301-304 \\
\hline & & 34 & $39-50$ & 300 \\
\hline & & 34 & & $4500-5600$ \\
\hline & & 42 & $55-72$ & $2601-3003$ \\
\hline 6200 & Phoenix, AZ & 4 & $8-9$ & 101-116 \\
\hline \multirow[t]{3}{*}{6282} & Pittsburgh-Beaver Valley PA (C) & 42 & $22-34$ & $1301-1400$ \\
\hline & & 42 & & $3301-3303$ \\
\hline & & 42 & & $3800-3902$ \\
\hline \multirow[t]{2}{*}{6442} & Portland-Vancouver, OR-WA (C) & 41 & $2-7$ & $1000-1500$ \\
\hline & & 53 & 10 & 1901-1902 \\
\hline \multirow[t]{3}{*}{6483} & Providence-Pawtucket-Woonsocket, RI-MA & 25 & $34-35$ & 4000 \\
\hline & & 25 & & 4200 \\
\hline & & 44 & $1-7$ & $100-800$ \\
\hline 6520 & Provo-Orem, UT & 49 & 6 & 500 \\
\hline \multirow[t]{2}{*}{6640} & Raleigh-Durham, NC & 37 & $15-19$ & $2301-2500$ \\
\hline & & 37 & & 2700 \\
\hline 6680 & Reading, PA & 42 & $49-50$ & $3101-3102$ \\
\hline \multirow[t]{2}{*}{6720} & Reno, NV & 32 & $3-4$ & 100 \\
\hline & & 32 & & $300-400$ \\
\hline \multirow[t]{2}{*}{6760} & Richmond-Petersburg, VA & 51 & $22-25$ & $1800-2100$ \\
\hline & & 51 & & 2400 \\
\hline 6840 & Rochester, NY & 36 & $19-22$ & $1900-2200$ \\
\hline 6880 & Rockford, IL & 17 & $2-3$ & $300-400$ \\
\hline \multirow[t]{3}{*}{6920} & Sacramento, CA & 6 & $7-11$ & 700 \\
\hline & & 6 & & $1000-1200$ \\
\hline & & 6 & & 2801-2906 \\
\hline \multirow[t]{2}{*}{6960} & Saginaw-Bay City-Midland, MI & 26 & $8-9$ & $800-900$ \\
\hline & & 26 & 11 & 1100 \\
\hline \multirow[t]{2}{*}{7040} & St. Louis, MO-IL & 17 & $18-22$ & $1700-2100$ \\
\hline & & 29 & $22-26$ & $1101-1500$ \\
\hline
\end{tabular}


Table 2, continued

\begin{tabular}{|c|c|c|c|c|}
\hline MSA & C/MSA Name & $\begin{array}{l}\text { State } \\
\text { Num. }\end{array}$ & $\begin{array}{c}1980 \\
\text { County } \\
\text { Group } \\
\end{array}$ & $\begin{array}{c}1990 \\
\text { PUMA }\end{array}$ \\
\hline 7120 & Salinas-Seaside-Monterey, CA & 6 & 33 & $3800-3902$ \\
\hline 7160 & Salt City-Ogden, UT & 49 & $1-5$ & $100-400$ \\
\hline 7240 & San Antonio, TX & 48 & $40-42$ & $4401-4600$ \\
\hline 7320 & San Diego CA & 6 & $51-52$ & $3301-3313$ \\
\hline \multirow[t]{4}{*}{7362} & San Francisco-Oakland-San Jose, CA (C) & 6 & $12-19$ & 900 \\
\hline & & 6 & $30-32$ & $1300-2206$ \\
\hline & & 6 & & $3401-3411$ \\
\hline & & 6 & & 3600 \\
\hline 7480 & Santa Barbara-Santa Maria-Lompoc, CA & 6 & 35 & $3201-3202$ \\
\hline 7510 & Sarasota, FL & 12 & 28 & $3101-3102$ \\
\hline \multirow[t]{3}{*}{7560} & Scranton-Wilkes-Barre, PA & 42 & $7-8$ & $500-700$ \\
\hline & & 42 & $10-15$ & 1800 \\
\hline & & 42 & & 3200 \\
\hline \multirow[t]{3}{*}{7602} & Seattle-Tacoma, WA (C) & 53 & $13-14$ & $1001-1004$ \\
\hline & & 53 & $17-19$ & $1301-1304$ \\
\hline & & 53 & & $1701-1808$ \\
\hline 7680 & Shreveport, LA & 22 & $1-2$ & 100 \\
\hline 7840 & Spokane, WA & 53 & 5 & $500-600$ \\
\hline 8000 & Springfield, MA & 25 & $2-6$ & $200-700$ \\
\hline 8120 & Stockton, CA & 6 & $20-21$ & $2301-2304$ \\
\hline \multirow[t]{2}{*}{8160} & Syracuse, NY & 36 & $13-16$ & 400 \\
\hline & & 36 & & $1400-1700$ \\
\hline \multirow[t]{2}{*}{8280} & Tampa-St. Petersburg-Clearwater, FL & 12 & 11 & 4000 \\
\hline & & 12 & $21-26$ & $4200-4500$ \\
\hline \multirow[t]{2}{*}{8400} & Toledo, $\mathrm{OH}$ & 39 & $1-4$ & $100-200$ \\
\hline & & 39 & & $3601-3604$ \\
\hline 8520 & Tucson, AZ & 4 & $5-6$ & $201-205$ \\
\hline \multirow[t]{2}{*}{8560} & Tulsa, OK & 40 & $4-7$ & 100 \\
\hline & & 40 & & $600-700$ \\
\hline 8680 & Utica-Rome, NY & 36 & $10-12$ & $501-600$ \\
\hline 8780 & Visalia-Tulare-Porterville, CA & 6 & 26 & 3500 \\
\hline
\end{tabular}


Table 2, continued

\begin{tabular}{|c|c|c|c|c|}
\hline MSA & C/MSA Name & $\begin{array}{l}\text { State } \\
\text { Num. }\end{array}$ & $\begin{array}{l}1980 \\
\text { County } \\
\text { Group } \\
\end{array}$ & $\begin{array}{c}1990 \\
\text { PUMA } \\
\end{array}$ \\
\hline \multirow{7}{*}{\multicolumn{2}{|c|}{8840 Washington, DC-MD-VA }} & 11 & 1 & $101-105$ \\
\hline & & 24 & 3 & 400 \\
\hline & & 24 & $9-11$ & 700 \\
\hline & & 24 & & 900 \\
\hline & & 24 & & $1201-1307$ \\
\hline & & 51 & $27-31$ & $800-1100$ \\
\hline & & 51 & & 2200 \\
\hline 8960 & West Palm Beach-Boca Raton-Delray FL & 12 & $33-35$ & 2901-2906 \\
\hline 9040 & Wichita, KS & 20 & $4-6$ & $400-600$ \\
\hline 9240 & Worcester, MA & 25 & $7-9$ & $800-1100$ \\
\hline \multirow[t]{2}{*}{9280} & York PA & 42 & $38-40$ & 2300 \\
\hline & & 42 & & $2501-2503$ \\
\hline \multirow[t]{2}{*}{9320} & Youngstown-Warren, $\mathrm{OH}$ & 39 & $19-22$ & $4801-4802$ \\
\hline & & 39 & & 4901-4902 \\
\hline
\end{tabular}

NOTES: (C) indicates 1990 CMSA. 
Table 3: Population and Relative Population of Metropolitan Areas Using 1980 and 1990 Definitions

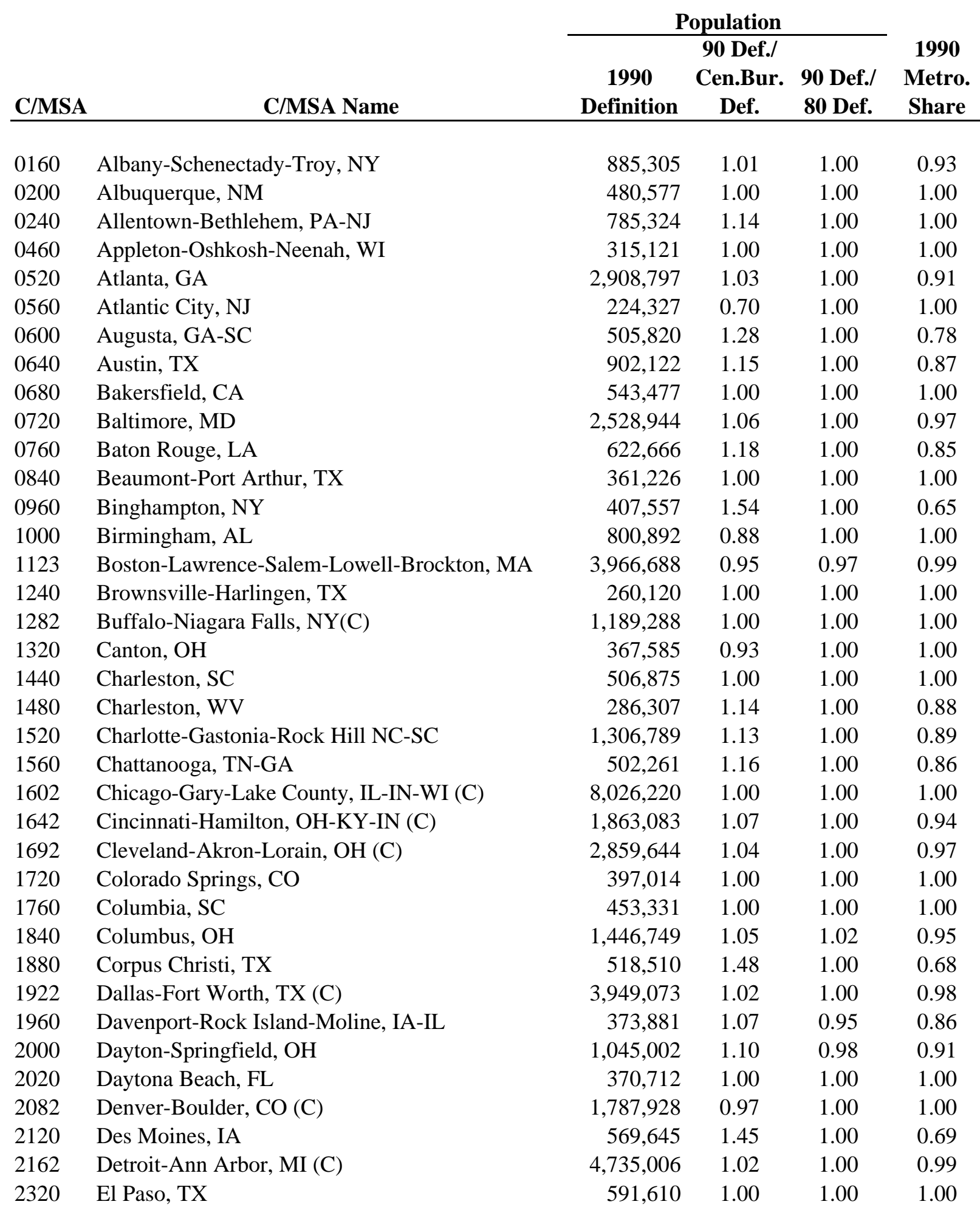


Table 3, continued

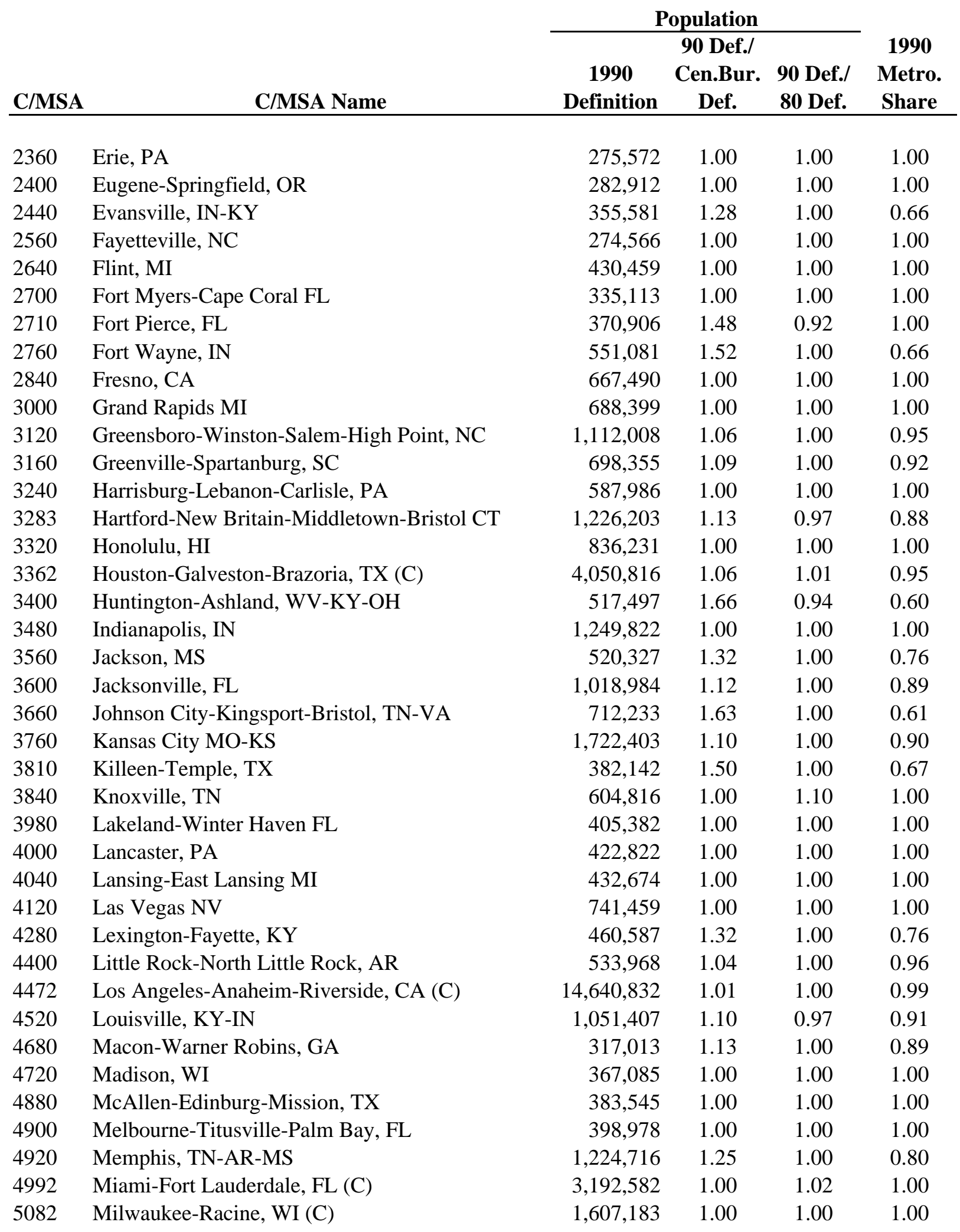


Table 3, continued

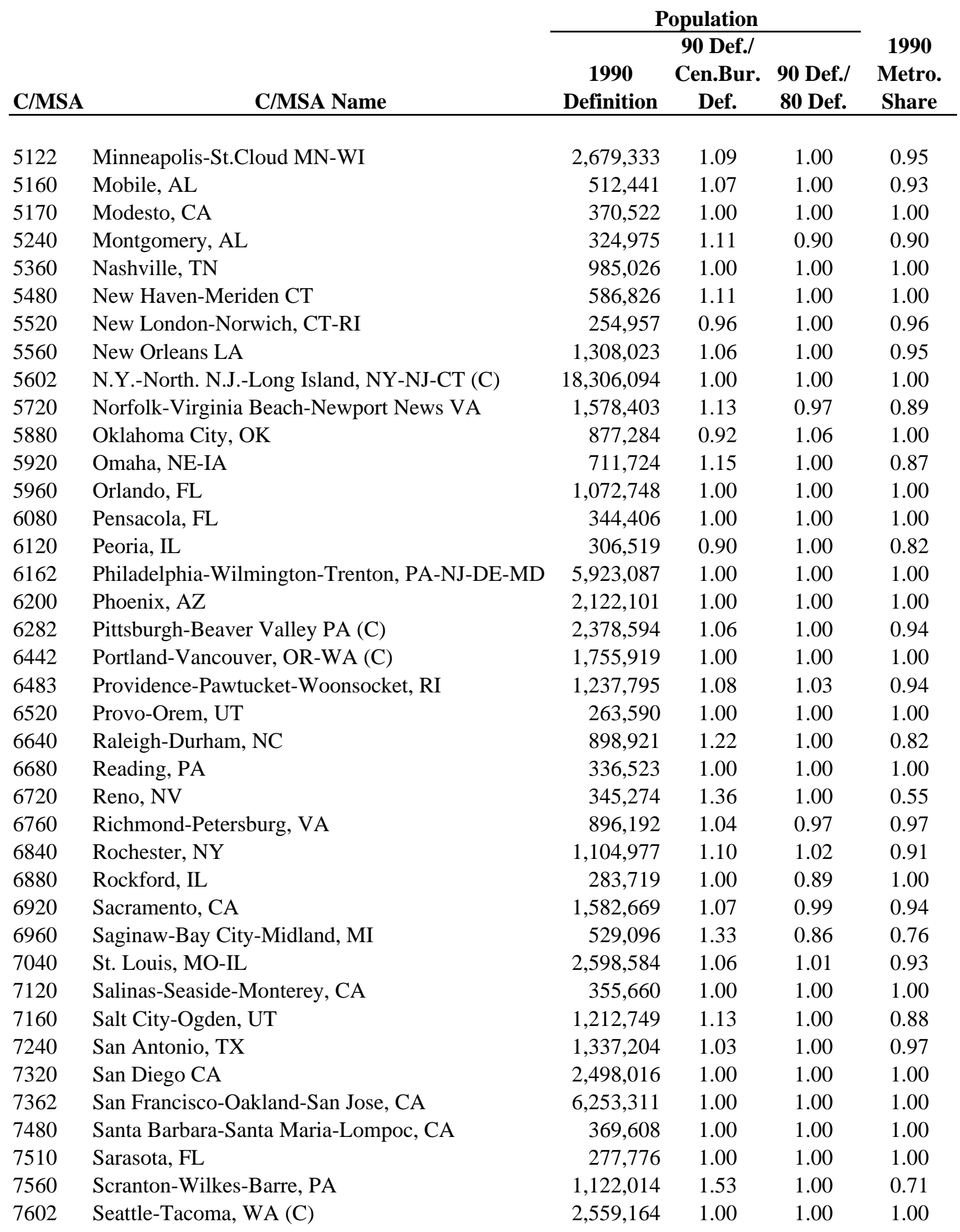


Table 3, continued

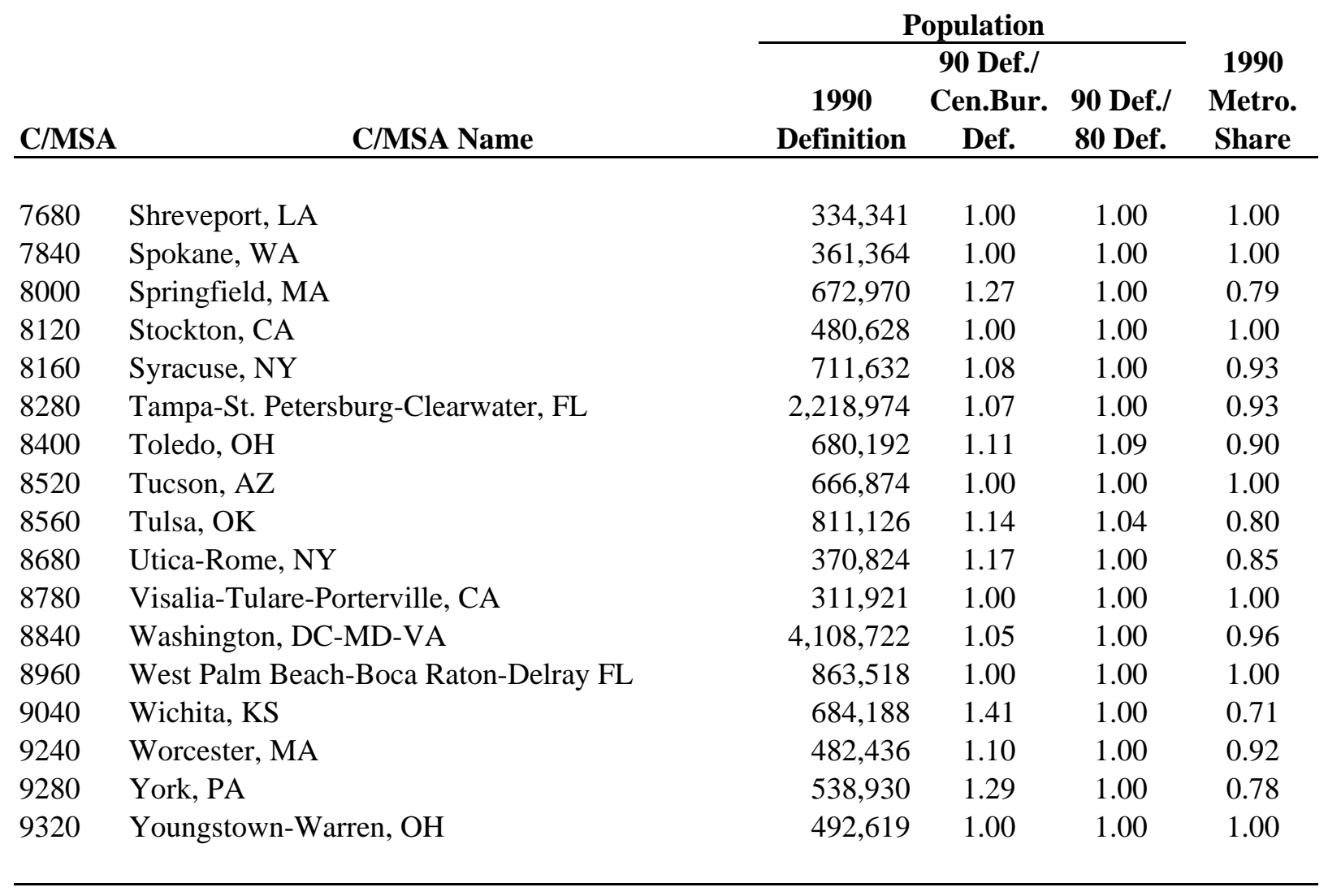

NOTES: See text for description of column headings. 
Table 4: 1970-1980-1990 Matches Based on 1970 MSA Definitions

\begin{tabular}{|c|c|c|c|c|c|c|c|}
\hline & MSA & $\begin{array}{l}1970 \text { Counties/ } \\
\text { Other Counties }\end{array}$ & $\begin{array}{l}\text { Cnty } \\
\text { Num } \\
\end{array}$ & $\begin{array}{l}1980 \\
\text { Pop. }\end{array}$ & $\begin{array}{l}1970 \\
\text { County } \\
\text { Group }\end{array}$ & $\begin{array}{l}1980 \\
\text { County } \\
\text { Group } \\
\end{array}$ & $\begin{array}{c}1990 \\
\text { PUMA } \\
\end{array}$ \\
\hline \multicolumn{8}{|c|}{ Alabama } \\
\hline \multirow[t]{2}{*}{1} & Birmingham & $\begin{array}{l}\text { Jefferson } \\
\text { Walker } \\
\text { Shelby } \\
\text { St Clair } \\
\text { Blount } \\
\text { Franklin* } \\
\text { Winston* }\end{array}$ & $\begin{array}{l}01: 073 \\
01: 127 \\
01: 117 \\
01: 115 \\
01: 009 \\
01: 059 \\
01: 133\end{array}$ & $\begin{array}{r}617,324 \\
68,660 \\
66,298 \\
41,205 \\
36,459 \\
28,350 \\
21,953\end{array}$ & $\begin{array}{l}4101 \\
4101 \\
4101\end{array}$ & $\begin{array}{c}\text { 009, 010 } \\
008 \\
011 \\
011 \\
008\end{array}$ & $\begin{array}{c}\text { 01801-01806 } \\
00200 \\
01700 \\
01700 \\
00200 \\
00200\end{array}$ \\
\hline & $\begin{array}{l}\text { *these two cour } \\
\text { No Match: } 198\end{array}$ & $\begin{array}{l}\text { are included with } 3 \text { oth } \\
\mathbf{1 0 . 3 2 \%} \text { more than } 1\end{array}$ & $\begin{array}{l}\text { aties in } 19 \\
\text { ad } 19901\end{array}$ & s $12.16 \% \mathrm{~m}$ & re than 1 & & \\
\hline \multirow[t]{2}{*}{2} & Mobile & $\begin{array}{l}\text { Mobile } \\
\text { Baldwin } \\
\quad \text { Escambia }\end{array}$ & $\begin{array}{l}01: 097 \\
01: 003 \\
01: 053\end{array}$ & $\begin{array}{r}364,980 \\
78,556 \\
38,440\end{array}$ & $\begin{array}{l}12201 \\
12201\end{array}$ & $\begin{array}{c}\text { 019, } 020 \\
021 \\
021\end{array}$ & $\begin{array}{l}00700 \\
00800 \\
00800\end{array}$ \\
\hline & 1980 and 1990 & ch with $8.67 \%$ more $t$ & 70 & & & & \\
\hline \multicolumn{8}{|c|}{ Arkansas } \\
\hline 3 & $\begin{array}{l}\text { Little Rock } \\
\text { N. Little Rock }\end{array}$ & $\begin{array}{l}\text { Saline } \\
\text { Pulaski }\end{array}$ & $\begin{array}{l}05: 125 \\
05: 119\end{array}$ & $\begin{array}{r}53,161 \\
340,613\end{array}$ & $\begin{array}{l}10401 \\
10401\end{array}$ & $\begin{array}{c}010 \\
008,009 \\
010\end{array}$ & $\begin{array}{l}01500 \\
01500\end{array}$ \\
\hline \multicolumn{8}{|c|}{ Arizona } \\
\hline 4 & Phoenix & Maricopa & 04:013 & $1,509,052$ & 14101 & 008, 009 & 00101-00116 \\
\hline 5 & Tuscon & Pima & 04:019 & 513,443 & 14102 & 005,006 & $00201-00205$ \\
\hline \multicolumn{8}{|c|}{ California } \\
\hline 6 & $\begin{array}{l}\text { Anaheim } \\
\text { Santa Ana } \\
\text { Garden Grove }\end{array}$ & Orange & 06:059 & $1,932,709$ & 14302 & $\begin{array}{l}043,044, \\
045,046\end{array}$ & $\begin{array}{c}\text { 04200, 04300, 04400, } \\
\text { 04500, 04600, 04700, } \\
\text { 04801-04808 }\end{array}$ \\
\hline 7 & Bakersfield & Kern & 06:029 & 403,089 & 14303 & 036, 037 & $04900,05001-05002$ \\
\hline 8 & Fresno & Fresno & 06:019 & 514,621 & 14304 & 027, 028 & 04000,04100 \\
\hline 9 & $\begin{array}{l}\text { Los Angeles } \\
\text { Long Beach }\end{array}$ & Los Angeles & 06:037 & $7,477,503$ & 14301 & $\begin{array}{l}\text { 040, 041, } \\
\quad 042\end{array}$ & $\begin{array}{c}\mathbf{0 5 2 0 0 ,}, 05300,05400, \\
05500,05600,05700, \\
05800,05900,06000, \\
0610006200,06300, \\
06401-06424, \\
\text { 06501-06521, 06600 }\end{array}$ \\
\hline 10 & $\begin{array}{l}\text { Oxnard } \\
\text { Ventura }\end{array}$ & Ventura & $06: 111$ & 529,174 & 14305 & 038, 039 & $06701-06705$ \\
\hline \multirow[t]{2}{*}{11} & Sacramento & $\begin{array}{l}\text { Yolo } \\
\text { Sacramento }\end{array}$ & $\begin{array}{l}06: 113 \\
06: 067\end{array}$ & $\begin{array}{l}113,374 \\
783,381\end{array}$ & $\begin{array}{l}14501 \\
14501\end{array}$ & $\begin{array}{c}011 \\
008,009\end{array}$ & $\begin{array}{c}01000 \\
\text { 02801, 02802, } 02803 \\
\mathbf{0 2 9 0 1 - 0 2 9 0 6}\end{array}$ \\
\hline & & Placer & 06:061 & 117,247 & 14501 & 010 & 01100 \\
\hline
\end{tabular}


Table 4, continued

\begin{tabular}{|c|c|c|c|c|c|c|}
\hline MSA & $\begin{array}{l}1970 \text { Counties/ } \\
\text { Other Counties }\end{array}$ & $\begin{array}{l}\text { Cnty } \\
\text { Num } \\
\end{array}$ & $\begin{array}{l}1980 \\
\text { Pop. }\end{array}$ & $\begin{array}{c}1970 \\
\text { County } \\
\text { Group }\end{array}$ & $\begin{array}{c}1980 \\
\text { County } \\
\text { Group } \\
\end{array}$ & $\begin{array}{c}1990 \\
\text { PUMA } \\
\end{array}$ \\
\hline \multicolumn{7}{|l|}{ Calfornia, continued } \\
\hline $\begin{array}{l}12 \text { Salinas } \\
\text { Monterey }\end{array}$ & Monterey & $06: 053$ & 290,444 & 14701 & $\mathbf{0 3 3}$ & 03800, 03901-03902 \\
\hline $\begin{array}{l}13 \text { San Bernardino } \\
\text { Riverside } \\
\text { Ontario }\end{array}$ & $\begin{array}{l}\text { Riverside } \\
\text { San Bernardino } \\
\quad \text { Imperial }\end{array}$ & $\begin{array}{l}06: 065 \\
06: 071\end{array}$ & $\begin{array}{r}663,166 \\
895,016 \\
92,110\end{array}$ & $\begin{array}{l}14306 \\
14307\end{array}$ & $\begin{array}{c}049,050 * \\
047,048 \\
050\end{array}$ & $\begin{array}{c}\text { 06800, 06901-06905 } \\
07000,07100 \\
07201-07207\end{array}$ \\
\hline $\begin{array}{l}* 492,290 \text { of } \mathrm{Riv} \\
\mathbf{1 9 7 0} \text { and } \mathbf{1 9 9 0}\end{array}$ & $\begin{array}{l}\text { de is in county group } 050 \\
\text { ch, } 1980 \text { has } \mathbf{5 . 9 1 \% ~ m o}\end{array}$ & & & & & \\
\hline 14 San Diego & San Diego & 06:073 & $1,861,846$ & 14201 & 051,052 & 03301-03313 \\
\hline $\begin{array}{l}15 \text { San Francisco } \\
\text { Oakland }\end{array}$ & $\begin{array}{l}\text { San Francisco } \\
\text { Alameda } \\
\text { Contra Costa } \\
\text { Marin } \\
\text { San Mateo }\end{array}$ & $\begin{array}{l}06: 075 \\
06: 001 \\
06: 013 \\
06: 041 \\
06: 081\end{array}$ & $\begin{array}{r}678,974 \\
1,105,379 \\
656,380 \\
222,568 \\
587,329\end{array}$ & $\begin{array}{l}14704 \\
14702 \\
14703 \\
14703 \\
14705\end{array}$ & $\begin{array}{c}014 \\
016,017 \\
018 \\
019 \\
015\end{array}$ & $\begin{array}{c}\text { 01901-01906 } \\
\text { 02000, 02101-02109 } \\
\text { 01700, 01801-01805 } \\
\text { 01501-01502 } \\
\text { 02201-02206 }\end{array}$ \\
\hline 16 San Jose & Santa Clara & 06:085 & $1,295,071$ & 14706 & 030, 031 & 03401-03411 \\
\hline 17 Santa Barbara & Santa Barbara & 06:083 & 298,694 & 14308 & 035 & 03201-03202 \\
\hline 18 Stockton & San Joaquin & 06:077 & 347,342 & 14401 & 020,021 & 02301-02304 \\
\hline \multicolumn{7}{|l|}{ Colorado } \\
\hline 19 Denver & $\begin{array}{l}\text { Adams } \\
\text { Arapahoe } \\
\text { Boulder } \\
\text { Jefferson } \\
\text { Denver }\end{array}$ & $\begin{array}{l}08: 001 \\
08: 005 \\
08: 013 \\
08: 059 \\
08: 031\end{array}$ & $\begin{array}{l}245,944 \\
293,621 \\
189,625 \\
371,753 \\
492,365\end{array}$ & $\begin{array}{l}13101 \\
13101 \\
13102 \\
13102 \\
13103\end{array}$ & $\begin{array}{c}007 \\
008,009 \\
010 \\
011,012 \\
006\end{array}$ & $\begin{array}{c}\text { 00201, 00301-00302 } \\
\text { 00201-00202, } 00400 \\
\text { 00701-00702 } \\
\text { 00500, 00601-00602 } \\
\text { 00101-00104 }\end{array}$ \\
\hline \multicolumn{7}{|l|}{ Connecticut } \\
\hline 20 Bridgeport & $\begin{array}{l}\text { New Haven (part) } \\
\text { Litchfield (part) }\end{array}$ & $\begin{array}{l}\text { 09:009 } \\
09: 005\end{array}$ & $\begin{array}{r}44,819 \\
1,654\end{array}$ & 1311 & $\begin{array}{c}\text { 001, 002, } \\
003,004, \\
005,006 \\
006\end{array}$ & $\begin{array}{c}\text { 01100, } 01200,01300, \\
01400,01500,01600, \\
01700 \\
01200\end{array}$ \\
\hline
\end{tabular}

No match, 1980 has $6.38 \%$ more than 1970,1990 has $0.24 \%$ more than 1970

\begin{tabular}{|c|c|c|c|c|c|c|}
\hline 21 Hartford & Hartford & 09:003 & 806,350 & 501 & $\begin{array}{c}\text { 015, 016, } \\
017,018, \\
019,020 \\
021\end{array}$ & $\begin{array}{c}00200,00300,00400 \\
0050000600,00700 \\
00800\end{array}$ \\
\hline & Tolland (part) & 09:013 & $27,974 *$ & & 018 & \\
\hline & Tolland (part) & 09:013 & $39,426^{*}$ & & 019 & \\
\hline & Litchfield (part) & 09:005 & $10,732 *$ & & 021 & \\
\hline
\end{tabular}

*county group 018 has 119,522 of county 003 , county group 019 has 80,693 of county 003 , and county group 021 has 99,909 of county 003

1970 and 1990 match, 1980 has $9.69 \%$ more

\begin{tabular}{|c|c|c|c|c|c|c|}
\hline 22 New Haven & New Haven & 09:009 & 559,232 & 503 & $\begin{array}{c}006^{*}, \\
\text { 007, 008, } \\
\mathbf{0 0 9 , 0 1 0} \\
006\end{array}$ & $\begin{array}{l}\text { 01800, } 01900,02000 \\
\text { 02100, } 02200,02300 \\
\text { 02400 }\end{array}$ \\
\hline
\end{tabular}

$* 44,819$ of county 009 is in county group 006 . 1970 and 1990 match, 1980 has $8.01 \%$ less 
Table 4, continued

\begin{tabular}{|c|c|c|c|c|c|c|}
\hline MSA & $\begin{array}{l}1970 \text { Counties/ } \\
\text { Other Counties }\end{array}$ & $\begin{array}{l}\text { Cnty } \\
\text { Num } \\
\end{array}$ & $\begin{array}{l}1980 \\
\text { Pop. }\end{array}$ & $\begin{array}{l}1970 \\
\text { County } \\
\text { Group } \\
\end{array}$ & $\begin{array}{l}1980 \\
\text { County } \\
\text { Group } \\
\end{array}$ & $\begin{array}{c}1990 \\
\text { PUMA } \\
\end{array}$ \\
\hline \multicolumn{7}{|l|}{ Delaware } \\
\hline 23 Wilmington & $\begin{array}{l}\text { Cecil, MD } \\
\text { New Castle, DE } \\
\text { Salem, NJ } \\
\text { Caroline, MD } \\
\text { Kent } \\
\text { Queen Anne's } \\
\text { Talbot } \\
\text { Cape May, NJ }\end{array}$ & $\begin{array}{l}24: 015 \\
10: 003 \\
34: 033 \\
24: 011 \\
24: 029 \\
24: 035 \\
24: 041 \\
34: 009\end{array}$ & $\begin{array}{r}60,430 \\
398,115 \\
64,676 \\
23,143 \\
16,695 \\
25,508 \\
25,604 \\
82,266\end{array}$ & $\begin{array}{l}1411 \\
1411 \\
1411\end{array}$ & $\begin{array}{l}\text { 24:012 } \\
\text { 10:001 } \\
34: 039 \\
24: 012 \\
24: 012 \\
24: 012 \\
24: 012 \\
34: 039\end{array}$ & $\begin{array}{c}\text { 24:00500 } \\
\text { 10:00301-00304 } \\
34: 00300 \\
24: 00500 \\
24: 00500 \\
24: 00500 \\
24: 00500 \\
34: 00300\end{array}$ \\
\hline \multicolumn{7}{|l|}{ District of Columbia } \\
\hline 24 Washington, DC & $\begin{array}{l}\text { DC } \\
\text { Montgomery, MD } \\
\text { Prince George,s, MD } \\
\text { Alexandia City, VA } \\
\text { Arlington, VA } \\
\text { Fairfax City, VA } \\
\text { Falls Church City, VA } \\
\text { Fairfax, VA } \\
\text { Laudon, VA } \\
\text { Prince William, VA } \\
\quad \text { Manassas City, VA } \\
\quad \text { Manasses Park City,V }\end{array}$ & $\begin{array}{l}11: 001 \\
24: 031 \\
24: 033 \\
51: 510 \\
51: 013 \\
51: 600 \\
51: 610 \\
51: 059 \\
51: 107 \\
51: 153 \\
51: 683 \\
51: 685\end{array}$ & $\begin{array}{r}638,333 \\
579,053 \\
665,071 \\
103,217 \\
152,599 \\
19,390 \\
9,515 \\
596,901 \\
57,427 \\
144,703 \\
15,438 \\
6,524\end{array}$ & $\begin{array}{l}1604 \\
1605 \\
1606 \\
1607 \\
1607 \\
1608 \\
1608 \\
1608 \\
1608 \\
1608 \\
* \\
*\end{array}$ & $\begin{array}{l}\text { 11:001 } \\
\text { 24:009 } \\
\text { 24:010 } \\
51: 030 \\
51: 029 \\
51: 031 \\
51: 031 \\
51: 031 \\
51: 028 \\
51: 028 \\
51: 028 \\
51: 028\end{array}$ & $\begin{array}{c}\text { 00101-00105 } \\
01201-01206 \\
01301-01307 \\
01000 \\
00800 \\
01100 \\
01100 \\
01100 \\
00900 \\
00900 \\
00900 \\
00900\end{array}$ \\
\hline
\end{tabular}

*these two counties may be included in the 1970 county groups here. they do not appear individually on the 1970 map.

\section{Florida}

\begin{tabular}{|c|c|c|c|c|c|c|c|}
\hline 25 & $\begin{array}{l}\text { Fort Lauderdale } \\
\text { Hollywood }\end{array}$ & Broward & 12:011 & $1,018,200$ & 3301 & $\begin{array}{c}\text { 036, 037, } \\
038,039 \\
040,041 \\
042\end{array}$ & $\begin{array}{c}\text { 03200, } 03300,03400 \\
03501-03506\end{array}$ \\
\hline 26 & Jacksonville & Duval & $12: 031$ & 571,003 & 3101 & 007 & 01000 \\
\hline \multirow[t]{4}{*}{27} & Miami & Dade & $12: 025$ & $1,625,802$ & 3302 & $\begin{array}{c}\text { 043, 044, } \\
045,046, \\
047,048, \\
049,050 \\
\text { 051, 052, } \\
053 *\end{array}$ & $\begin{array}{c}\text { 03601-03602, } \\
370003800 \\
03901-03909\end{array}$ \\
\hline & & Monroe & $12: 087$ & 63,188 & & 053* & 02400 \\
\hline & & Collier & $12: 021$ & 85,971 & & & 02400 \\
\hline & $\begin{array}{l}* \text { county group } 053 \\
1970 \text { and } 1990 \mathrm{~m}\end{array}$ & $\begin{array}{l}\text { ontains } 149,67 \\
\text { h. } 1980 \text { has } 3\end{array}$ & & & & & \\
\hline \multirow[t]{3}{*}{28} & Orlando & $\begin{array}{l}\text { Seminole } \\
\text { Orange }\end{array}$ & $\begin{array}{l}12: 117 \\
12: 095\end{array}$ & $\begin{array}{l}179,752 \\
471,016\end{array}$ & $\begin{array}{l}3201 \\
3201\end{array}$ & $\begin{array}{c}019 \\
017,018\end{array}$ & $\begin{array}{c}02200,02300 \\
01600,01700,01800 \\
01900,02000\end{array}$ \\
\hline & & $\begin{array}{l}\text { Osceola } \\
\text { Brevard }\end{array}$ & $\begin{array}{l}12: 097 \\
12: 009\end{array}$ & $\begin{array}{r}49,287 \\
272,959\end{array}$ & $\begin{array}{l}3202 \\
3202\end{array}$ & 019 & 02100 \\
\hline & 1970 and $1990 \mathrm{~m}$ & h. 1980 has 7 & & & & & \\
\hline \multirow[t]{2}{*}{29} & Tampa & Hillsborough & 12:057 & 646,960 & 3303 & 022,023 & 04500 \\
\hline & St. Petersburg & Pinellas & $12: 103$ & 728,531 & 3304 & $\begin{array}{l}024,025 \\
026\end{array}$ & 04400 \\
\hline 30 & West Palm Beach & Palm Beach & 12:099 & 576,863 & 3305 & $\begin{array}{l}\text { 033, 034, } \\
\quad 035\end{array}$ & 02901-02906 \\
\hline
\end{tabular}


Table 4, continued

\begin{tabular}{ccccccc} 
& & \multicolumn{4}{c}{1970} & 1980 \\
& & & & \\
MSA & Other Counties/ & Cnty & 1980 & County & County & 1990 \\
Group & Group & PUMA \\
\hline
\end{tabular}

Georgia

31 Atlanta

\begin{tabular}{lrr} 
Dekalb & $13: 089$ & 483,024 \\
Fulton & $13: 121$ & 589,904 \\
Clayton & $13: 063$ & 150,357 \\
Cobb & $13: 067$ & 297,718 \\
Gwinnett & $13: 135$ & 166,903 \\
$\quad$ Douglas & $13: 097$ & 54,573 \\
$\quad$ Rockdale & $13: 247$ & 36,747 \\
$\quad$ Henry & $13: 151$ & 36,309 \\
\multicolumn{1}{c}{ Fayette } & $13: 113$ & 29,043
\end{tabular}

\begin{tabular}{|cc|}
\hline 4001 & 004,006 \\
4002 & 004,005 \\
4003 & 009 \\
4003 & 007 \\
4003 & 008 \\
& 007 \\
& 008 \\
\hline
\end{tabular}

01901-01904

31 Atlanta

(n)

1970 and 1990 match. 1980 has $5.41 \%$ more

32 Augusta

Aiken, SC
Richmond, GA
Columbia,GA
McDuffie, GA
ch. 1990 has $20.42 \%$ more

Hawaii

33 Honolulu

Honolulu
Kauii
Mauii
Hawaii
$\quad$ Kalawao

$\begin{array}{rr}45: 003 & 105,625 \\ 13: 245 & 181,629 \\ 13: 073 & 40,114 \\ 13: 189 & 18,546\end{array}$

1970 and 1980 match. 1990 has $20.42 \%$ more

$15: 007$
$15: 009$
$15: 001$
$15: 005$

39,082

70,847

92,053

144

$\begin{array}{ll}2901 & 45: 016 \\ 2901 & 13: 026\end{array}$

45:016
$13: 026$

001,002

003

003

003

003

1980 and 1990 match with $0.01 \%$ more than 1970

\section{Iowa}

34 Davenport, IA
Rock Island, Ill
Moline, Ill
17:073

$17: 161$

19:163

17:095

$17: 131$

$17: 155$

17:011
Bureau, IL

1980 and 1990 match with $15.10 \%$ less than 1970

35 Des Moines

Polk

19:153

303,170

Illinois

36 Chicago

Cook

Du Page

Kane

Lake

McHenry

Will

37 Peoria

Woodford

Peoria

Tazewell

Bureau

Fulton

Stark

Putnam

Marshall

Mason

$17: 031$
$17: 043$
$17: 089$
$17: 097$
$17: 111$
$17: 197$
$17: 203$
$17: 143$
$17: 179$
$17: 011$
$17: 057$
$17: 175$
$17: 155$
$17: 123$
$17: 125$

$5,253,655$

658,835

278,405

440,372

147,897

324,460

33,320

200,466

132,078

39,114

43,687

7,389

6,085

14,479

19,492

$\begin{array}{rrr}\mathbf{7 4 0 1} & 17: 010 & 00600 \\ \mathbf{7 4 0 1} & \mathbf{1 7 : 0 1 1} & \mathbf{0 1 0 0 0} \\ \mathbf{7 4 0 1} & \mathbf{1 9 : 0 1 6} & \mathbf{0 1 4 0 0} \\ & 17: 010 & 17: 00600 \\ & & 17: 00600 \\ & & 17: 00600\end{array}$

$9301 \quad 008,009$

00700, 00800

$\mathbf{7 2 0 1}$
$\mathbf{7 2 0 2}$
$\mathbf{7 2 0 3}$
$\mathbf{7 2 0 4}$
$\mathbf{7 2 0 5}$
$\mathbf{7 2 0 5}$
$\mathbf{7 3 0 1}$
$\mathbf{7 3 0 1}$
$\mathbf{7 3 0 1}$

032, 033

03001-03019, 03101-03114

034 03201-03206

035 03501-03502

036 03401-03404

$037 \quad 03300$

038

007

008

009

007

007

007

007

007
03700, 03800, 03900

00700

00800

00900

00700

00700

00700

00700

1980 and 1990 match with $9.11 \%$ less than 1970 
Table 4, continued

\begin{tabular}{ccccccc}
\multicolumn{1}{c}{ MSA } & $\begin{array}{c}\text { 1970 Counties/ } \\
\text { Other Counties }\end{array}$ & $\begin{array}{c}\text { Cnty } \\
\text { Num }\end{array}$ & $\begin{array}{c}\mathbf{1 9 8 0} \\
\text { Pop. }\end{array}$ & $\begin{array}{c}\text { 1970 } \\
\text { County } \\
\text { Group }\end{array}$ & $\begin{array}{c}\text { 1980 } \\
\text { County } \\
\text { Group }\end{array}$ & $\begin{array}{c}\text { 1990 } \\
\text { PUMA }\end{array}$ \\
\hline \multirow{2}{*}{ Illinois, continued } & & & & & & \\
38 Rockford & Winnebago & $17: 201$ & 250,884 & $\mathbf{7 6 0 1}$ & $\mathbf{0 0 2 , 0 0 3}$ & $\mathbf{0 0 3 0 0 , 0 0 4 0 0}$ \\
& Boone & $17: 007$ & 28,630 & $\mathbf{7 6 0 1}$ & 004 & $\mathbf{0 0 4 0 0}$ \\
& De Kalb & $17: 037$ & 74,624 & & 004 &
\end{tabular}

1970 and 1990 match. 1980 has $10.24 \%$ less.

Indiana

$\begin{array}{ll}39 \text { Fort Wayne } & \text { Allen } \\ 40 \text { Gary } & \text { Lake } \\ \text { Hammond } & \text { Porter } \\ \text { East Chicago } & \\ 41 \text { Indianapolis } & \text { Marion } \\ & \text { Boone } \\ & \text { Hamilton } \\ & \text { Hendricks } \\ & \text { Morgan } \\ & \text { Hancock } \\ & \text { Johnson } \\ & \text { Shelby } \\ & \text { St Joseph } \\ & \text { Marshall } \\ & \text { Kosciusko } \\ & \text { Jasper } \\ & \text { Newth Bend } \\ & \text { Pulaski } \\ & \text { Starke }\end{array}$

1980 and 1990 match with $13.95 \%$ less than 1970

Kansas

43 Wichit

Butler
Sedgwick
Wabaunsee
Lyon
Morris
Dickenson
Greenwood
Coffey
Marion
Chase

$\begin{array}{lr}20: 015 & 44,782 \\ 20: 173 & 366,531 \\ 20: 197 & 6,867 \\ 20: 111 & 35,108 \\ 20: 127 & 6,419 \\ 20: 041 & 20,175 \\ 20: 073 & 8,764 \\ 20: 031 & 9,370 \\ 20: 115 & 13,522 \\ 20: 017 & 3,309\end{array}$

1980 and 1990 match with $10.89 \%$ less than 1970

Kentucky

44 Louisville

Floyd, IN
Clark, IN
Jefferson, KY

$\quad$ Harrison, IN
Washington, IN
Scott, IN

$\begin{array}{rr}18: 043 & 61,169 \\ 18: 019 & 88,838 \\ 21: 111 & 685,004 \\ & \\ 18: 061 & 27,276 \\ 18: 175 & 21,932 \\ 18: 143 & 20,422\end{array}$

5001

5001

18:034

21:003

21:004

18:034

18:034

18:035
01700, 01800

00800, 00900, 01000

01200

00101-00107

03300

03500

03300

03400

03500

03400

03500

01500, 01600

02800

02800

1970 and 1990 match. 1980 has $8.34 \%$ more 
Table 4, continued

\begin{tabular}{|c|c|c|c|c|c|c|}
\hline MSA & $\begin{array}{l}1970 \text { Counties/ } \\
\text { Other Counties }\end{array}$ & $\begin{array}{l}\text { Cnty } \\
\text { Num } \\
\end{array}$ & $\begin{array}{l}1980 \\
\text { Pop. }\end{array}$ & $\begin{array}{c}1970 \\
\text { County } \\
\text { Group } \\
\end{array}$ & $\begin{array}{c}1980 \\
\text { County } \\
\text { Group } \\
\end{array}$ & $\begin{array}{c}1990 \\
\text { PUMA } \\
\end{array}$ \\
\hline \multicolumn{7}{|l|}{ Louisiana } \\
\hline 45 Baton Rouge & East Baton Rouge & $22: 033$ & 366,191 & 12301 & 015,016 & $01301-01302,01400$ \\
\hline \multirow[t]{5}{*}{46 New Orleans } & Orleans Parish & 22:071 & 557,515 & 12302 & 021 & 01901-01904 \\
\hline & Jefferson Parish & $22: 051$ & 454,592 & 12303 & $\begin{array}{l}022,023, \\
024,024, \\
025\end{array}$ & $02000,02100,02200$ \\
\hline & St. Bernard Parish & 22:087 & 64,097 & 12303 & 025 & 02300 \\
\hline & St. Tammany Parish & $22: 103$ & 110,869 & 12303 & 026 & 02300 \\
\hline & Plaquemines Parish & $22: 075$ & 26,049 & & 025 & 02300 \\
\hline \multicolumn{7}{|c|}{1980 and 1990 match. 1970 has $2.19 \%$ less } \\
\hline \multirow[t]{3}{*}{47 Shreveport } & Caddo & $22: 015$ & 80,721 & 11601 & 001,002 & 00100 \\
\hline & Bossier & $22: 017$ & 252,358 & 11601 & 001,002 & 00100 \\
\hline & Webster Parish & $22: 119$ & 43,631 & & 002 & \\
\hline
\end{tabular}

1970 and 1990 match. 1980 has $13.10 \%$ more

Maryland

48 Baltimore

Baltimore City
Baltimore
Ann Arundel
Carroll
Harford
Howard

786,775

1601

006

655,615

370,775

24:003

96,356

24:013

145,930

24:025

118,572

1602

1603

1603

1603

1603

004

008

004

005

007

01501-01504
00301-00306
00201-00204
00600
01000
01100

Massachusetts

49 Boston

Suffolk
Essex

25:025

650,142

406

019, 021, 022

402

013, 014 ,

015, 016,

018, 019

Middlesex

$1,367,034$

403

011, 012,

017,018

020, 021 ,

023-027 010

Norfolk

25:021

606,587

404

023, 026,

027, 028 ,

029030 ,

031, 032 ,

34

02001-02005, 02100

01600, 01700, 01800, 01900, 02700

01400, 01500, 02100 , 02200, 02300, 02400, 02500, 02800, 02900, 03000, 03100, 03200

02500, 02600, 03100, 03300, 03400, 03500, 03600, 03800

$$
04200
$$

$\begin{array}{lcc}\text { Plymouth } & 25: 023 & 405,43 \\ & & \\ \text { Worcester } & 25: 027 & * \\ \text { (part) } & * * \\ \text { Bristol } & 25: 005 & * * \\ & \text { (part) } & \end{array}$
405
031, 032, 033 036, 037
010
026
032
034, 036 037

03600, 03700, 03800, 04100, 04300

03800 ****

$04100 * * *, 04200 * * *$ $04300 * * *$

* in county group, 010 , county 027 has 161,208 while 017 has 27,783 , in 026027 has 29,583 while others have 178,072

** in county group 032 , county 005 has 16,623 of 136,697 , in county group 034 it has 101,273 of 107,130 , in county group 036 it has 153,965 of 169,425 , and in county group 037 it has 56,817 of 103,845

*** in PUMA 03800, county 005 has 19,807 of 149,515 , in PUMA 04100 it has 83,832 of $1,094,84$, in PUMA 04200 it has 101691 of 108,562 , and in PUMA 04300 it has 59,172 of 103,261

No match. 1980 is missing $2.62 \%$ of 1970 and has additions of $1.26 \% .1990$ is missing $2.09 \%$ of 1970 and has additional $0.54 \%$. 
Table 4, continued

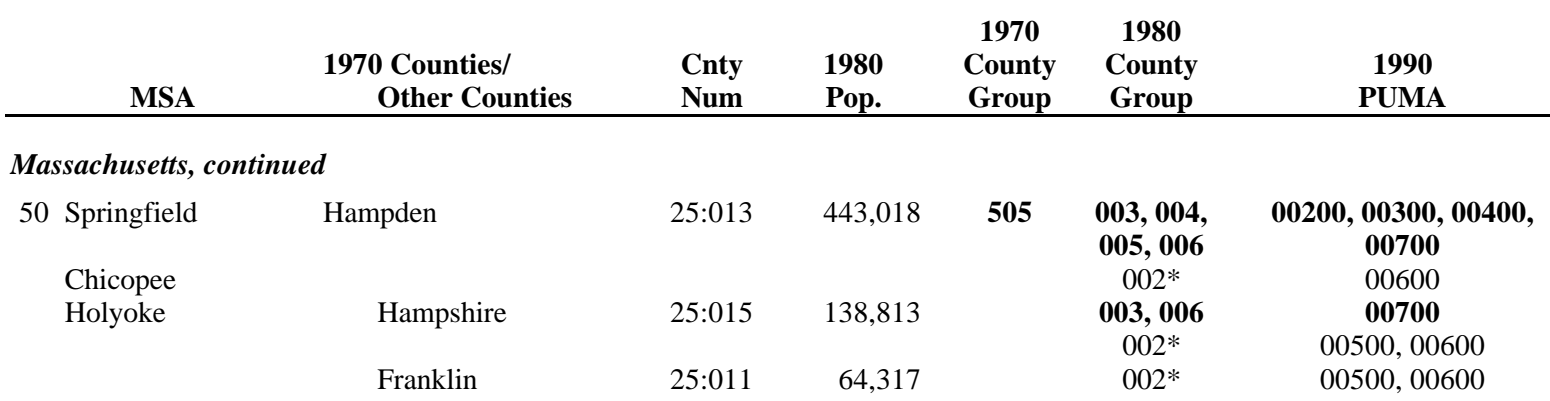

*in county group 002 , county 011 has 64,317 of 127,730 , county 013 has 10,891 . In county group 003 , county 103 has 70,118 of 122,166 . In county group 006 , county 013 has 82,858 of 117,101 in PUMA 00600 , county 013 has 6,512 of 106,403 . In PUMA 00700 , county 013 has 91,580 of 102,159 .

No match. 1980 has $2.45 \%$ less than 1970 in one area and $19.48 \%$ more in another. 1990 has $1.39 \%$ less and $2.39 \%$ more.

51 Worcester

\begin{tabular}{|c|c|c|c|c|c|}
\hline Worcester & $25: 027$ & 614,239 & 407 & $\begin{array}{l}007,008, \\
009,010\end{array}$ & $\begin{array}{l}\text { 00800, 00900, 01000 } \\
01100,01200,01300\end{array}$ \\
\hline Middlesex (part) & $\begin{array}{c}25: 017 \\
\text { (part) }\end{array}$ & * & & 010 & 01300 \\
\hline
\end{tabular}

*in county group 010 , county 017 has 27,783 of 188,991 In PUMA01300, county 017 has 31,713 of 105,881 . Probably different parts of county 017 so no match.

No match. 1980 has $4.52 \%$ more than 1970 in one area and 1990 has about $5.16 \%$ more (maybe in another area).

\section{Michigan}

52 Detroit

\section{Wayne}

$26: 163$

$2,337,891$

Macomb

Oakland

53 Flint

Genesee
Lapeer
$\quad$ Shiawassee

$\begin{array}{rrrc}26: 099 & 694,600 & \mathbf{6 6 0 2} & \mathbf{0 4 5 - 0 4 9} \\ 26: 125 & 1,011,793 & \mathbf{6 6 0 3} & \mathbf{0 5 0 - 0 5 7} \\ & & & \\ 26: 049 & 450,449 & \mathbf{6 6 0 4} & \mathbf{0 2 1 , 0 2 2} \\ & & & \mathbf{0 2 3} \\ 26: 087 & 70,038 & \mathbf{6 6 0 4} & 024 \\ 26: 155 & 71,140 & & 024\end{array}$

03301-03308, 03401-03405, 03500, 03600,03700 03800, 03901-03903, 04000 04101-04107, 04200

02000, 02101-02102 02200 02200

1980 and 1990 match with $13.46 \%$ less than 1970 ( or they can have $13.67 \%$ more)

$\begin{array}{llrrrrr}54 \text { Grand Rapids } & \text { Ottawa } & 26: 139 & 157,174 & \mathbf{6 8 0 1} & \mathbf{0 1 7} & \mathbf{0 1 5 0 0} \\ & \text { Kent } & 26: 081 & 444,506 & \mathbf{6 8 0 1} & \mathbf{0 1 3 , 0 1 4 ,} & \mathbf{0 1 3 0 0 , \mathbf { 0 1 4 0 1 } - 0 1 4 0 2} \\ & & & & & & \\ 55 \text { Lansing } & \text { Clinton } & 26: 037 & 55,893 & \mathbf{6 9 0 1} & \mathbf{0 2 0} & \mathbf{0 1 9 0 0} \\ & \text { Eaton } & 26: 045 & 88,337 & \mathbf{6 9 0 1} & \mathbf{0 2 0} & \mathbf{0 1 9 0 0} \\ & \text { Ingham } & 26: 065 & 275,520 & \mathbf{6 9 0 1} & \mathbf{0 1 8 , 0 1 9} & \mathbf{0 1 7 0 0 , 0 1 8 0 0}\end{array}$

\section{Minnesota}

\begin{tabular}{|c|c|c|c|c|c|c|}
\hline 56 Duluth, MN & St Louis, MN & $27: 137$ & 222,229 & 8001 & $\begin{array}{l}27: 003 \\
27: 004\end{array}$ & 00300 \\
\hline \multirow{10}{*}{ Superior, WI } & Douglas, WI & $55: 031$ & 44,421 & 8001 & 55:001 & 00100 \\
\hline & Ashland, WI & $55: 003$ & 16,783 & & 55:001 & 00100 \\
\hline & Bayfield & $55: 007$ & 13,822 & & 55:001 & 00100 \\
\hline & Burnett & $55: 013$ & 12,340 & & 55:001 & 00100 \\
\hline & Iron & $55: 051$ & 6,730 & & $55: 001$ & 00100 \\
\hline & Price & $55: 099$ & 15,788 & & 55:001 & 00100 \\
\hline & Rusk & $55: 107$ & 15,589 & & 55:001 & 00100 \\
\hline & Sawyer & $55: 113$ & 12,843 & & 55:001 & 00100 \\
\hline & Tayor & $55: 119$ & 18,817 & & 55:001 & 00100 \\
\hline & Wahburn & $55: 129$ & 13,174 & & 55:001 & 00100 \\
\hline
\end{tabular}

1980 and 1990 match with $16.66 \%$ less than 1970

$\mathbf{0 0 3 0 0}$
00100
00100
00100
00100
00100
00100
00100
00100
00100
00100 
Table 4, continued

\begin{tabular}{|c|c|c|c|c|c|c|}
\hline MSA & $\begin{array}{l}1970 \text { Counties/ } \\
\text { Other Counties }\end{array}$ & $\begin{array}{l}\text { Cnty } \\
\text { Num } \\
\end{array}$ & $\begin{array}{l}1980 \\
\text { Pop. }\end{array}$ & $\begin{array}{c}1970 \\
\text { County } \\
\text { Group } \\
\end{array}$ & $\begin{array}{c}1980 \\
\text { County } \\
\text { Group } \\
\end{array}$ & $\begin{array}{c}1990 \\
\text { PUMA } \\
\end{array}$ \\
\hline \multicolumn{7}{|c|}{ Minnesota, continued } \\
\hline \multirow{5}{*}{$\begin{array}{l}57 \text { Minneapolis } \\
\text { St. Paul }\end{array}$} & Anoka & 27:003 & 195,998 & 8203 & 024 & 01200,01300 \\
\hline & Dakota & $27: 037$ & 194,279 & 8203 & 022 & 02300,02400 \\
\hline & Hennepin & $27: 053$ & 941,411 & 8201 & 014,015 & $\begin{array}{l}01500,01600,01700 \\
01800,01900,02000\end{array}$ \\
\hline & Ramsey & $27: 123$ & 459,784 & 8202 & 019,020 & 02100,02200 \\
\hline & Washington & $27: 163$ & 113,571 & 8203 & 023 & 01400 \\
\hline \multicolumn{7}{|l|}{ Mississippi } \\
\hline \multirow[t]{4}{*}{58 Jackson } & Hinds & 28:049 & 250,998 & 12001 & 009 & 00900 \\
\hline & Rankin & $28: 121$ & 69,427 & 12001 & 010 & 01000 \\
\hline & Copiah & $28: 029$ & 26,503 & & 010 & 01000 \\
\hline & Simpson & $28: 127$ & 23,441 & & 010 & 01000 \\
\hline
\end{tabular}

1980 and 1990 match with $15.59 \%$ more than 1970

Missouri

$\begin{array}{lrr}\text { Jackson, MO } & 29: 095 & 629,266 \\ & & \\ & & \\ \text { Johnson, KS } & 20: 091 & 270,269 \\ \text { Cass,MO } & 29: 037 & 51,029 \\ \text { Wyandotte, KS } & 20: 209 & 172,335 \\ \text { Clay, MO } & 29: 047 & 136,488 \\ \text { Platte, MO } & 29: 165 & 46,341 \\ \quad & & \\ \quad \text { Ray, MO } & 29: 177 & 21,378 \\ \quad \text { Lafayette, MO } & 29: 107 & \end{array}$

$9803 \quad 29: 008$,

29:009,

29:010,

29:012

$9801220: 009$

$980129: 012$

9802 20:010

$9802 \quad 29: 008$

29:011

$9802 \quad 29: 008$

29:011

29:012

01001-01005

00901-00903
00900
01000
00800
00800

00900
00900

1980 has $1.64 \%$ more than 1970.1990 has about $2 \%$ more than 1980

60 St. Louis

Madison, IL
St. Clair, IL
St Louis
St Louis County
Franklin
Jefferson
St Charles

$\begin{array}{rr}17: 119 & 247,691 \\ & \\ 17: 163 & 267,531 \\ & \\ 29: 510 & 453,085 \\ 29: 189 & 973,896 \\ 29: 071 & 71,233 \\ 29: 099 & 146,183 \\ 29: 183 & 144,107\end{array}$

10101

17:019

17:020

10102

17:021

17:022

$10103 \quad 29: 023$

10104 29:025

10105

$*$

10105

29:026

10105 29:024
01800, 01900
02000, 02100
01201-01203
01101-01104
01400
01400
01300

* in 1980 Frankilin is with 6 other counties.

1970 and 1990 match. 1980 has 3.19\% less.

Nebraska

\begin{tabular}{|c|c|c|c|c|c|c|}
\hline \multirow[t]{11}{*}{61 Omaha } & Douglas,NE & $31: 055$ & 397,038 & 9401 & 006 & 01001-01004 \\
\hline & Sarpy, NE & $31: 153$ & 86,015 & 9401 & 007 & 00900 \\
\hline & Pottawattamie, IA & $19: 155$ & 86,561 & 9401 & 019 & 01700 \\
\hline & Cass,IA & 19:029 & $*$ & & 019 & 01700 \\
\hline & Fremont, IA & 19:071 & $*$ & & 019 & 01700 \\
\hline & Harrison, IA & 19:085 & $*$ & & 019 & 01700 \\
\hline & Mills, IA & $19: 129$ & $*$ & & 019 & 01700 \\
\hline & Montgomery, IA & $19: 137$ & $*$ & & 019 & 01700 \\
\hline & Page, IA & $19: 145$ & * & & 019 & 01700 \\
\hline & Shelby, IA & $19: 165$ & $*$ & & 019 & 01700 \\
\hline & Washington, NE & $31: 177$ & 15,508 & & 007 & 00900 \\
\hline
\end{tabular}

*together these counties total 103,606 in 1980 population.

1980 and 1990 match with $2.72 \%$ more than 1970 in one area and $15.20 \%$ less in another.

Nevada
62 Las Vegas
Clark
$32: 003$
463,087
14001
001,002
00201-00205 
Table 4, continued

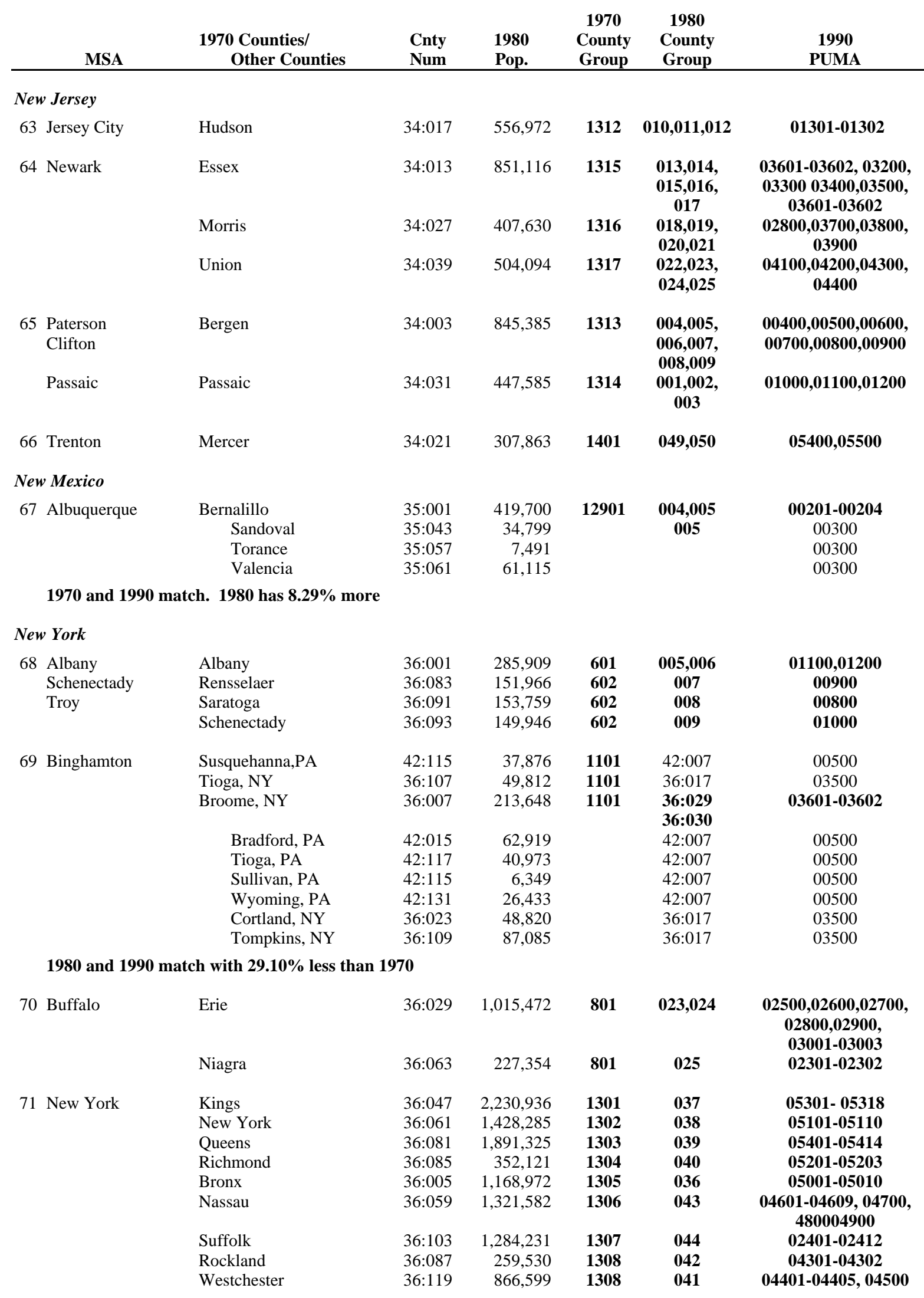


Table 4, continued

\begin{tabular}{clccccc} 
MSA & $\begin{array}{c}\text { 1970 Counties/ } \\
\text { Other Counties }\end{array}$ & $\begin{array}{c}\text { Cnty } \\
\text { Num }\end{array}$ & $\begin{array}{c}\mathbf{1 9 8 0} \\
\text { Pop. }\end{array}$ & $\begin{array}{c}\text { 1970 } \\
\text { County } \\
\text { Group }\end{array}$ & $\begin{array}{c}\text { 1980 } \\
\text { County } \\
\text { Group }\end{array}$ & $\begin{array}{c}\text { 1990 } \\
\text { PUMA }\end{array}$ \\
\hline \multirow{2}{*}{ New York, continued } & & & & & & \\
72 Rochester & & & & & & \\
& Orleans & $36: 073$ & 38,496 & $\mathbf{7 0 1}$ & 022 & 02200 \\
& Monroe & $36: 055$ & 702,238 & $\mathbf{7 0 1}$ & $\mathbf{0 1 9 , 0 2 0}$ & $\mathbf{0 2 0 0 0 , 0 2 1 0 0}$ \\
& Livingston & $36: 051$ & 57,006 & $\mathbf{7 0 1}$ & $\mathbf{0 2 1}$ & $\mathbf{0 1 9 0 0}$ \\
& Wayne & $36: 117$ & 84,581 & $\mathbf{7 0 1}$ & $\mathbf{0 2 1}$ & $\mathbf{0 1 9 0 0}$ \\
& Ontario & $36: 069$ & 88,909 & & $\mathbf{0 2 1}$ & $\mathbf{0 1 9 0 0}$ \\
& Yates & $36: 123$ & 21,459 & & $\mathbf{0 2 1}$ & 03300 \\
& Genesee & $36: 037$ & 59,400 & & 022 & 02200 \\
& Wyoming & $36: 121$ & 39,895 & & 022 & 02200 \\
& Steuben & $36: 101$ & 99,217 & & & 03300
\end{tabular}

No match. 1980 and 1990 have $4.36 \%$ less than 1970 in one area. 1980 has $12.51 \%$ and 1990 has $10.08 \%$ more in another area.

73 Syracuse

Oswego
Onondaga
Madison
$\quad$ Chenango

$\begin{array}{rr}36: 075 & 113,901 \\ 36: 067 & 463,920 \\ 36: 053 & 65,150 \\ 36: 017 & 49,344\end{array}$

702

702

702

015
013,014
016
016

$\begin{array}{rrrc}36: 065 & 253,466 & \mathbf{7 0 3} & \mathbf{0 1 1 , 0 1 2} \\ 36: 043 & 66,714 & \mathbf{7 0 3} & 010 \\ 36: 035 & 55,153 & & 010 \\ 36: 057 & 53,439 & & 010\end{array}$

1980 and 1990 match with $20.84 \%$ less than 1970 .

North Carolina

75 Charlotte

Mecklenburg
Union
Stanely

$37: 119$
$37: 179$
$37: 167$
404,270
70,380
48,517

2401

$\mathbf{0 3 6 , 0 3 7}$
$\mathbf{0 3 7}$
00801-00804
01200
01200

1970 and 1980 match. 1990 has $10.22 \%$ more.

76 Greensboro
Winston
Salem
High Point

Guilford
Forsyth
Randolph
Yadkin
$\quad$ Alamance
Surry
Stokes
Davie

$37: 081$

37:067

$37: 151$

$37: 197$

37:001

$37: 171$

$37: 169$

317,154

243,683

91,728

28,439

99,319

59,449

33,086

24,599

No match. 1980 has $17.65 \%$ less than 1970 . 1990 has $4.18 \%$ less.

\section{Ohio}

$\begin{array}{ll}77 \text { Akron } & \begin{array}{l}\text { Summit } \\ \text { Portage }\end{array} \\ 78 \text { Canton } & \text { Stark }\end{array}$

$\begin{array}{ll}39: 153 & 524,472 \\ 39: 133 & 135,856 \\ & \\ 39: 151 & 378,823\end{array}$

\begin{tabular}{cc|}
6301 & 023,024 \\
6301 & 025 \\
\hline 6302 & 027,028 \\
\hline
\end{tabular}

$\begin{array}{cc}\mathbf{2 3 0 1} & \mathbf{0 1 0 , 0 1 1} \\ \mathbf{2 3 0 2} & \mathbf{0 0 8 , 0 0 9} \\ \mathbf{2 3 0 2} & 013 \\ \mathbf{2 3 0 2} & 007 \\ & 013 \\ & 007 \\ & 007 \\ & 007\end{array}$

$\mathbf{0 1 3 0 0 , 0 1 4 0 0}$
$\mathbf{0 2 0 0 0 , 0 2 1 0 0}$
$\mathbf{0 1 6 0 0}$
02200
01700
02200
02200
02200

00600

00600 
Table 4, continued

\begin{tabular}{|c|c|c|c|c|c|c|}
\hline MSA & $\begin{array}{l}1970 \text { Counties/ } \\
\text { Other Counties }\end{array}$ & $\begin{array}{l}\text { Cnty } \\
\text { Num } \\
\end{array}$ & $\begin{array}{l}1980 \\
\text { Pop. }\end{array}$ & $\begin{array}{c}1970 \\
\text { County } \\
\text { Group } \\
\end{array}$ & $\begin{array}{c}1980 \\
\text { County } \\
\text { Group } \\
\end{array}$ & $\begin{array}{c}1990 \\
\text { PUMA } \\
\end{array}$ \\
\hline \multicolumn{7}{|l|}{ Ohio, continued } \\
\hline \multirow[t]{12}{*}{79 Cincinnati } & Hamilton & 39:061 & 873,224 & 5801 & $\begin{array}{c}\mathbf{0 5 2 , 0 5 3} \\
\mathbf{0 5 4}\end{array}$ & 05401-05406 \\
\hline & Clermont & $39: 025$ & 128,483 & 5802 & 055 & 03300 \\
\hline & Warren & $39: 165$ & 99,276 & 5802 & 055 & 03000 \\
\hline & Dearborn, IN & 18:029 & 34,291 & 5802 & $*$ & $*$ \\
\hline & Boone, KY & 21:015 & 45,842 & 5802 & 21:002 & 01400 \\
\hline & Campbell, KY & 21:037 & 83,317 & 5802 & 21:002 & 01400 \\
\hline & Kenton, KY & $21: 117$ & 137,058 & 5802 & 21:001 & 01300 \\
\hline & Carrol, KY & 21:041 & 9,270 & & 21:002 & 01400 \\
\hline & Gallatin, KY & 21:077 & 4,842 & & 21:002 & 01400 \\
\hline & Grant, KY & 21:081 & 13,308 & & 21:002 & 01400 \\
\hline & Owen. KY & $21: 187$ & 8,924 & & 21:002 & 01400 \\
\hline & Pendleton, KY & $21: 191$ & 10,989 & & 21:002 & 01400 \\
\hline
\end{tabular}

* with other counties that together have more people.

1980 and 1990 match with $2.45 \%$ less than 1970 in one area and $3.38 \%$ more in another.

80 Cleveland

$\begin{array}{lrr}\text { Cuyahoga } & 39: 035 & 1,498,410 \\ & & \\ & & \\ \text { Geauga } & 39: 055 & 74,474 \\ \text { Lake } & 39: 085 & 212,801 \\ \text { Medina } & 39: 103 & 113,150 \\ \quad \text { Ashtabula } & 39: 007 & 104,215\end{array}$

6304

008,009, 010,011 012,013, 014,015

6305

6305

017

6305

017

016

018
03901-03905, 04000, 04100 04200,04300,04400, 04500 00400 04600 00800 00400

1970 and 1980 match. 1990 has $3.92 \%$ less.

81 Columbus

$\begin{array}{lrr}\text { Franklin } & 39: 049 & 869,132 \\ \text { Delaware } & 39: 041 & 53,840 \\ \text { Pickaway } & 39: 129 & 43,662 \\ \text { Fairfield } & 39: 045 & 93,678 \\ \text { Madison } & 39: 097 & 33,004 \\ \quad \text { Union } & 39: 159 & 29,536 \\ \text { Ross } & 39: 141 & 65,004\end{array}$

$\begin{array}{cc}\mathbf{6 0 0 1} & \mathbf{4 2 0 4 3} \\ \mathbf{6 0 0 1} & 044 \\ \mathbf{6 0 0 1} & 044 \\ & 044 \\ & 044\end{array}$

05101-05107

01700

02700

044

01700

01700

02700

1980 and 1990 match with $10.09 \%$ less than 1970

82 Dayton

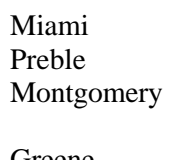

Darke

Lorain

Elyria

84 Toledo

85 Youngstown

Warren
Lorain

Monroe, MI

Lucas, $\mathrm{OH}$

Wood, $\mathrm{OH}$

Trumbull

Mahoning
39:109

$39: 135$

39:113

90,381

38,223

571,697

39:057 $\quad 129,769$

39:037 $\quad 55,096$

\begin{tabular}{cc}
$\mathbf{5 9 0 1}$ & $\mathbf{0 5 0}$ \\
$\mathbf{5 9 0 1}$ & $\mathbf{0 5 0}$ \\
$\mathbf{5 9 0 1}$ & $\mathbf{0 4 6 , 0 4 7}$, \\
& $\mathbf{0 4 8}$ \\
$\mathbf{5 9 0 1}$ & $\mathbf{0 4 9}$ \\
\hline
\end{tabular}

6306

39:093

(1)

26:115

39:095

134,659

471,741

39:173

107,372

39:155

39:099
241,863 289,487
006,007

26:033

39:002,

39:003

004

902019,020

902021,022
02100

02100 05201-05205

02600

02100

03700,03800

26:03000

39:03601-39:03604

00200

04801-04802

04901-04902 
Table 4, continued

\begin{tabular}{|c|c|c|c|c|c|c|}
\hline MSA & $\begin{array}{l}1970 \text { Counties/ } \\
\text { Other Counties }\end{array}$ & $\begin{array}{l}\text { Cnty } \\
\text { Num } \\
\end{array}$ & $\begin{array}{l}1980 \\
\text { Pop. }\end{array}$ & $\begin{array}{l}1970 \\
\text { County } \\
\text { Group } \\
\end{array}$ & $\begin{array}{c}1980 \\
\text { County } \\
\text { Group } \\
\end{array}$ & $\begin{array}{c}1990 \\
\text { PUMA } \\
\end{array}$ \\
\hline \multicolumn{7}{|l|}{ Oklahoma } \\
\hline \multirow[t]{6}{*}{86 Oklahoma City } & Canadian & 40:017 & 56,452 & 10701 & $\begin{array}{l}\mathbf{0 1 0} \\
013\end{array}$ & 00900 \\
\hline & Cleveland & 40:027 & 133,173 & 10701 & 010,012 & 01100 \\
\hline & Oklahoma & 40:109 & 568,933 & 10701 & 010,011 & 01000 \\
\hline & McClain & 40:087 & * & & $\begin{array}{l}\mathbf{0 1 0} \\
013\end{array}$ & \\
\hline & Pttawatomie & $40: 125$ & $*$ & & $\begin{array}{l}\mathbf{0 1 0} \\
013\end{array}$ & \\
\hline & Logan & $40: 083$ & 26,881 & & & 00900 \\
\hline
\end{tabular}

*123 of county 087 is in county group $010,20,168$ is in county group 013.51 of county 125 is in county group 010 and 55,188 is in county group $013.47,711$ of county 017 is in county group 013 .

No match. 1980 has $6.29 \%$ less than 1970 and 1990 has $3.54 \%$ more than 1970 .

$\begin{array}{llrrrrr}87 \text { Tulsa } & \text { Osage } & 40: 113 & 39,327 & \mathbf{1 0 6 0 1} & \mathbf{0 0 4 , 0 0 5} & \mathbf{0 0 6 0 0} \\ & \text { Tulsa } & 40: 143 & 470,593 & \mathbf{1 0 6 0 1} & \mathbf{0 0 4 , 0 0 5} & \mathbf{0 0 7 0 0} \\ & \text { Creek } & 40: 037 & 59,016 & \mathbf{1 0 6 0 1} & \mathbf{0 0 5} & \mathbf{0 0 6 0 0}\end{array}$

Oregon

88 Portland

$\begin{array}{lccccc}\text { Multnomah, OR } & 41: 051 & 562,640 & \mathbf{1 3 7 0 1} & \mathbf{0 0 2 , 0 0 3} & \mathbf{0 1 3 0 0 , 0 1 4 0 0} \\ \text { Clackamas, OR } & 41: 005 & 241,919 & \mathbf{1 3 7 0 2} & \mathbf{0 0 5} & \mathbf{0 1 3 0 0 , 0 1 5 0 0} \\ \text { Washington, OR } & 41: 067 & 245,808 & \mathbf{1 3 7 0 2} & \mathbf{0 0 4} & \mathbf{0 1 2 0 0 , 0 1 3 0 0} \\ \text { Clark, WA } & 53: 011 & 192,227 & \mathbf{1 3 7 0 2} & \mathbf{0 1 0} & \mathbf{0 1 9 0 1 - 0 1 9 0 2}\end{array}$

Pennsylvania

89 Allentown

Lehigh, PA

42:077

272,349

1413

051,052 , $\mathbf{0 5 3 , 0 5 4}$

Bethlehem

Northampton, PA

42:095

225,418

1414 052,053

Easton

$\begin{array}{crr}\text { Warren, NJ } & 34: 041 & 84,429 \\ \text { Hunterdon, NJ } & 34: 019 & 87,361 \\ \text { Sussex, NJ } & 34: 037 & 116,119\end{array}$

1414 054

34:051

34:051

34:052

02001-02002

02101-02102

34:04000

34:04000

1980 and 1990 match with $14.50 \%$ less than 1970 .

90 Erie

91 Harrisburg

92 Johnstown
42:049

42:043

42:041

42:099

42:021

42:111

42:009

42:057

42:061
279,780

232,317

178,541

35,718

183,263

81,243

46,784

12,842

42,253
901

1501

1501

1501

6201

6201
001,002

041,042

043,044

044

035

037

037

037

037

046-048

42:055

42:061-064

42:056-060

42:065-067

42:068-072

34:045-047

34:041-044

34:048
00101-00102

03601-03602

03700

03700

03400

03400

02400

02400

02400

1970 and 1990 match. 1980 has $30.71 \%$ less. (1980 and 1990 can also match)

$\begin{array}{llrrrrr}93 \text { Lancaster } & \text { Lancaster } & 42: 071 & 362,346 & \mathbf{1 5 0 2} & \mathbf{0 4 6 - 0 4 8} & \mathbf{0 3 5 0 1 - 0 3 5 0 3} \\ 94 \text { Philadelphia } & \text { Philadelphia } & 42: 101 & 1,688,210 & \mathbf{1 4 0 6} & \mathbf{4 2 : 0 5 5} & \mathbf{0 2 6 0 1 - 0 2 6 1 1} \\ & \text { Bucks } & 42: 017 & 479,211 & \mathbf{1 4 0 7} & \mathbf{4 2 : 0 6 1 - 0 6 4} & \mathbf{0 2 7 0 1 - 0 2 7 0 3} \\ & \text { Montgomery } & 42: 091 & 643,621 & \mathbf{1 4 0 8} & \mathbf{4 2 : 0 5 6 - 0 6 0} & \mathbf{0 2 8 0 1 - 0 2 8 0 5} \\ & \text { Chester } & 42: 029 & 316,660 & \mathbf{1 4 0 9} & \mathbf{4 2 : 0 6 5 - 0 6 7} & \mathbf{0 2 9 0 1 - 0 2 9 0 3} \\ & \text { Delaware } & 42: 045 & 555,007 & \mathbf{1 4 1 0} & \mathbf{4 2 : 0 6 8 - 0 7 2} & \mathbf{0 3 0 0 1 - 0 3 0 0 3} \\ & \text { Burlington, NJ } & 34: 005 & 362,542 & \mathbf{1 4 0 4} & \mathbf{3 4 : 0 4 5 - 0 4 7} & \mathbf{0 4 5 0 0 , 0 4 6 0 0 , 0 4 7 0 0} \\ & \text { Camden, NJ } & 34: 007 & 471,650 & \mathbf{1 4 0 5} & \mathbf{3 4 : 0 4 1 - 0 4 4} & \mathbf{0 4 8 0 0 , 0 4 9 0 0 , 0 5 0 0 0 ,} \\ & & & & \mathbf{0 5 1 0 0} \\ & \text { Gloucester, NJ } & 34: 015 & 199,917 & \mathbf{1 4 0 5} & \mathbf{3 4 : 0 4 8} & \mathbf{0 5 2 0 0 , 0 5 3 0 0}\end{array}$


Table 4, continued

\begin{tabular}{|c|c|c|c|c|c|c|}
\hline MSA & $\begin{array}{l}1970 \text { Counties/ } \\
\text { Other Counties }\end{array}$ & $\begin{array}{l}\text { Cnty } \\
\text { Num } \\
\end{array}$ & $\begin{array}{l}1980 \\
\text { Pop. }\end{array}$ & $\begin{array}{c}1970 \\
\text { County } \\
\text { Group } \\
\end{array}$ & $\begin{array}{c}1980 \\
\text { County } \\
\text { Group } \\
\end{array}$ & $\begin{array}{c}1990 \\
\text { PUMA } \\
\end{array}$ \\
\hline \multicolumn{7}{|c|}{ Pennsylvania, continued } \\
\hline 95 Pittsburgh & $\begin{array}{l}\text { Allegheny } \\
\text { Westmoreland } \\
\text { Beaver } \\
\text { Washington } \\
\quad \text { Lawrence* }\end{array}$ & $\begin{array}{l}42: 003 \\
42: 129 \\
42: 007 \\
42: 125 \\
42: 073\end{array}$ & $\begin{array}{r}1,450,085 \\
392,294 \\
204,441 \\
217,074 \\
107,150\end{array}$ & $\begin{array}{l}6202 \\
6203 \\
6204 \\
6204\end{array}$ & $\begin{array}{c}\mathbf{0 2 5 - 0 3 1} \\
\mathbf{0 3 2 , 0 3 3} \\
\mathbf{0 2 3} \\
\mathbf{0 2 4} \\
022\end{array}$ & $\begin{array}{c}\text { 01301-01312 } \\
\text { 03301-03303 } \\
\text { 03800 } \\
\text { 03901-03902 } \\
\text { 03800 }\end{array}$ \\
\hline
\end{tabular}

* in 1970 Lawrence is with 5 other counties in county group 9003.

1970 and 1980 match. 1990 has $4.7 \%$ more. (1980 and 1990 can also match)

$\begin{array}{llccccc}96 \text { Reading } & \text { Berks } & 42: 011 & 312,509 & \mathbf{1 4 1 2} & \mathbf{0 4 9 , 0 5 0} & \mathbf{0 3 1 0 1 - 0 3 1 0 2} \\ 97 \text { Wilkes Barre } & \text { Luzerne } & 42: 079 & 343,079 & \mathbf{1 2 0 1} & \mathbf{0 1 2 , 0 1 3 ,} & \mathbf{0 0 7 0 0} \\ \text { Hazelton } & & & & & \mathbf{0 1 4} & \\ & & & & & \mathbf{0 2 3 0 0} \\ 98 \text { York } & \text { Adams } & 42: 001 & 68,292 & \mathbf{1 5 0 3} & \mathbf{0 4 0} & \mathbf{0 2 5 0 1 - 0 2 5 0 3} \\ & \text { York } & 42: 133 & 312,963 & \mathbf{1 5 0 3} & \mathbf{0 3 9 , 0 4 0} & \mathbf{0 2 3 0 0}\end{array}$

1970 and 1980 match. 1990 has $29.80 \%$ more. (1980 and 1990 can also match)

Rhode Island

$\begin{array}{llrrrrr}99 \text { Providence } & \text { Bristol } & 44: 001 & 46,942 & \mathbf{4 0 9} & \mathbf{0 0 4} & \mathbf{0 0 5 0 0} \\ \text { (this is the whole } & \text { Kent } & 44: 003 & 154,163 & \mathbf{4 0 9} & \mathbf{0 0 3 , 0 0 5} * & \mathbf{0 0 4 0 0} \\ \begin{array}{l}\text { state of Rhode } \\ \text { Island) }\end{array} & \text { Providence } & 44: 007 & 571,349 & \mathbf{4 0 9} & \mathbf{0 0 1 , 0 0 2 ,} & \mathbf{0 0 1 0 0 , 0 0 2 0 0 , 0 0 3 0 0} \\ & & & & & \mathbf{0 0 3 , 0 0 4} * \boldsymbol{0 0 7 0 0 , 0 0 8 0 0} & \mathbf{0 0 7 0 0} \\ & & & & \mathbf{0 0 6} & \mathbf{0 0 5 0 0} \\ & \text { Newport } & 44: 005 & 81,383 & \mathbf{4 1 0} & \mathbf{0 0 4} & \mathbf{0 0 6 0 0}\end{array}$

$* 29,803$ of county 003 is in county group 005 with county 009 .

**46,942 of county 001 and 50,980 of county 007 are with county 005 in county group 004 .

Can match all if use whole state. (not MSA as defined in 1970)

South Carolina

$\begin{array}{llrrrcc}100 \text { Charleston } & \text { Charleston } & 45: 019 & 276,974 & \mathbf{2 9 0 2} & \mathbf{0 1 9 , 0 2 0} & \mathbf{0 1 2 0 1 - 0 1 2 0 2} \\ & \text { Berkeley } & 45: 015 & 94,727 & \mathbf{2 9 0 2} & \mathbf{0 2 1} & \mathbf{0 1 1 0 0} \\ & \text { Dorchester } & 45: 035 & 58,761 & & \mathbf{0 2 1} & \mathbf{0 1 1 0 0}\end{array}$

1980 and 1990 match with $15.81 \%$ more than 1970 .

$\begin{array}{llccccc}\text { 101 Columbia } & \text { Richland } & 45: 079 & 269,735 & \mathbf{2 7 0 1} & \mathbf{0 1 3 , 0 1 4} & \mathbf{0 1 6 0 1 - 0 1 6 0 2} \\ & \text { Lexington } & 45: 063 & 140,353 & \mathbf{2 7 0 1} & \mathbf{0 1 5} & \mathbf{0 1 7 0 0} \\ \text { 102 Greenville } & \text { Pickens } & 45: 077 & 79,292 & \mathbf{2 6 0 1} & \mathbf{0 0 2} & \mathbf{0 0 1 0 0} \\ & \text { Greenville } & 45: 045 & 287,913 & \mathbf{2 6 0 1} & \mathbf{0 0 3 , 0 0 4} & \mathbf{0 0 2 0 1 - 0 0 2 0 2} \\ & \text { Oconee } & 45: 073 & 48,611 & & \mathbf{0 0 2} & \mathbf{0 0 1 0 0}\end{array}$

1980 and 1990 match with $13.24 \%$ more than 1970 .

Tennesse

$\begin{array}{ccccccc}\text { 103 Chattanooga } & \text { Hamilton, TN } & 47: 065 & 287,740 & \mathbf{4 4 0 1} & \begin{array}{c}\mathbf{4 7 : 0 1 5}, \\ \mathbf{4 7 : 0 1 6}\end{array} & \mathbf{0 1 2 0 0 , 0 1 3 0 0} \\ & & & & \mathbf{1 3 : 0 0 1} & \mathbf{0 0 3 0 0} \\ & \text { Walker, GA } & 13: 295 & 56,470 & \mathbf{4 4 0 1} & \mathbf{1 3} & \mathbf{1 3 : 0 0 3 0 0} \\ \text { Catoosa, GA } & 13: 047 & 36,991 & & \mathbf{1 3 : 0 0 1} & \mathbf{1 3 : 0 0 3 0 0} \\ \text { Dade, GA } & 13: 083 & 12,318 & & \mathbf{1 3 : 0 0 1} & \mathbf{1 3}\end{array}$

1980 and 1990 match with $14.33 \%$ more than 1970 . 
Table 4, continued

\begin{tabular}{llccccc}
\multicolumn{1}{c}{ MSA } & $\begin{array}{c}\text { 1970 Counties/ } \\
\text { Other Counties }\end{array}$ & $\begin{array}{c}\text { Cnty } \\
\text { Num }\end{array}$ & $\begin{array}{c}\mathbf{1 9 8 0} \\
\text { Pop. }\end{array}$ & $\begin{array}{c}\text { 1970 } \\
\text { County } \\
\text { Group }\end{array}$ & $\begin{array}{c}\text { 1980 } \\
\text { County } \\
\text { Group }\end{array}$ & $\begin{array}{c}\text { 1990 } \\
\text { PUMA }\end{array}$ \\
\hline Tennessee, continued & & & & & & \\
104 Knoxville & Blount & $47: 009$ & 77,770 & $\mathbf{4 6 0 1}$ & $\mathbf{0 2 4}$ & $\mathbf{0 0 6 0 0}$ \\
& Knox & $47: 093$ & 319,694 & $\mathbf{4 6 0 1}$ & $\mathbf{0 2 2 , 0 2 3}$ & $\mathbf{0 0 8 0 0 , 0 0 9 0 0}$ \\
& Anderson & $47: 001$ & 67,346 & $\mathbf{4 6 0 1}$ & $\mathbf{0 2 4}$ & $\mathbf{0 0 7 0 0}$ \\
& Union & $47: 173$ & 11,707 & & $\mathbf{0 2 4}$ & $\mathbf{0 0 7 0 0}$ \\
& Granger & $47: 057$ & 16,751 & & & $\mathbf{0 0 7 0 0}$ \\
& Jefferson & $47: 089$ & 31,284 & & & $\mathbf{0 0 7 0 0}$ \\
& Sevier & $47: 155$ & 41,418 & & & $\mathbf{0 0 6 0 0}$
\end{tabular}

No match. 1980 has $2.52 \%$ more than 1970 and 1990 has $21.76 \%$ more than 1970 .

\begin{tabular}{|c|c|c|c|c|c|c|}
\hline \multirow[t]{2}{*}{105 Memphis } & Shelby & $47: 157$ & 777,113 & 4201 & \multirow[t]{2}{*}{$\begin{array}{l}\text { 47:001, } \\
47: 002\end{array}$} & \multirow[t]{2}{*}{01900,02000} \\
\hline & Crittenden, AR & 05:035 & 49,499 & 4201 & & \\
\hline \multicolumn{7}{|c|}{1980 and 1990 match with $5.99 \%$ less than 1970 . } \\
\hline 106 Nashville & Wilson & 47:189 & 56,064 & 4501 & 011 & 02300 \\
\hline \multirow[t]{8}{*}{ Davidson } & Sumner & $47: 165$ & 85,790 & 4501 & 010 & 02400 \\
\hline & Davidson & 47:037 & 477,811 & 4501 & 009 & 00501-00505 \\
\hline & Robertson & $47: 147$ & 37,021 & & 010 & 02300 \\
\hline & Rutherford & $47: 149$ & 84,058 & & 011 & 02500 \\
\hline & Wilson & $47: 189$ & 56,064 & & & 02300 \\
\hline & Dickson & 47:043 & 30,037 & & 008 & 02300 \\
\hline & Williamson & $47: 187$ & 58,108 & & 008 & 02300 \\
\hline & Cheatham & $47: 021$ & 21,616 & & 008 & 02300 \\
\hline
\end{tabular}

No match. 1990 has $9.05 \%$ less than 1970.1980 is missing this $9.05 \%$ but also has and additional $5.97 \%$ of 1970 .

Texas

\begin{tabular}{|c|c|c|c|c|c|c|}
\hline 107 Austin & Travis & $48: 453$ & 419,573 & 11501 & 044 & 04901-04904, 05000 \\
\hline 108 Beaumont & Orange & $48: 361$ & 83,838 & 12501 & 056 & 06100 \\
\hline Port Arthur & Jefferson & $48: 245$ & 250,938 & 12501 & $\mathbf{0 5 5 , 0 5 4}$ & 05900,06000 \\
\hline & Hardin & $48: 199$ & 40,721 & & 056 & 06100 \\
\hline \multicolumn{7}{|c|}{1980 and 1990 match with $12.16 \%$ more than 1970} \\
\hline \multirow[t]{13}{*}{109 Corpus Christi } & San Patrico & $48: 409$ & 58,013 & 12702 & 035 & 04800 \\
\hline & Nueces & $48: 355$ & 268,215 & 12702 & 039 & 04301-04302 \\
\hline & Aransas & 48:007 & 14,260 & & 035 & 04800 \\
\hline & Bee & 48:025 & 26,030 & & 035 & 04800 \\
\hline & Brooks & 48:047 & 8,428 & & 035 & 04800 \\
\hline & Duval & $48: 131$ & 12,517 & & 035 & 04800 \\
\hline & Jim Wells & $48: 249$ & 36,498 & & 035 & 04800 \\
\hline & Kenedy & $48: 261$ & 543 & & 035 & 04800 \\
\hline & Kleberg & $48: 273$ & 33,358 & & 035 & 04800 \\
\hline & Live Oak & $48: 297$ & 9,606 & & 035 & 04800 \\
\hline & McMullen & $48: 311$ & 789 & & 035 & 04800 \\
\hline & Refugio & $48: 391$ & 9,289 & & 035 & 04800 \\
\hline & Willacy & $48: 489$ & 17,495 & & 035 & 04800 \\
\hline
\end{tabular}

1980 and 1990 match with $17.78 \%$ less than 1970 . 
Table 4, continued

\begin{tabular}{|c|c|c|c|c|c|c|}
\hline MSA & $\begin{array}{l}1970 \text { Counties/ } \\
\text { Other Counties }\end{array}$ & $\begin{array}{l}\text { Cnty } \\
\text { Num }\end{array}$ & $\begin{array}{l}1980 \\
\text { Pop. }\end{array}$ & $\begin{array}{c}1970 \\
\text { County } \\
\text { Group } \\
\end{array}$ & $\begin{array}{c}1980 \\
\text { County } \\
\text { Group } \\
\end{array}$ & $\begin{array}{c}1990 \\
\text { PUMA } \\
\end{array}$ \\
\hline \multicolumn{7}{|l|}{ Texas, continued } \\
\hline \multirow[t]{8}{*}{110 Dallas } & Collin & 48:085 & 144,576 & 11301 & 022 & 02300,02400 \\
\hline & Denton & $48: 121$ & 143,126 & 11301 & 023 & 02201-02202 \\
\hline & Rockwall & $48: 397$ & 14,528 & 11301 & 024 & 01500 \\
\hline & Kaufman & $48: 257$ & 39,015 & 11301 & 024 & 01500 \\
\hline & Dallas & $48: 113$ & $1,556,390$ & 11301 & 013 - 018 & $\begin{array}{c}\text { 02501-02509, } 02600 \\
\text { 02700 02800, } \\
\text { 02901-02904 }\end{array}$ \\
\hline & Tarrant* & $48: 439$ & 860,880 & 11302 & $\begin{array}{l}019,020 \\
021\end{array}$ & $\begin{array}{l}\text { 01901-01904, } \\
\text { 02001-02002, } \\
\text { 02101-02104 }\end{array}$ \\
\hline & Johnson & $48: 251$ & 67,649 & 11302 & & \\
\hline & Ellis & $48: 139$ & 59,743 & 11301 & 024 & 01500 \\
\hline \multicolumn{7}{|c|}{$\begin{array}{l}\text { *Fort Worth is also in Tarrant County } \\
1980 \text { and } 1990 \text { match with } 2.4 \% \text { more in } 1970 .\end{array}$} \\
\hline 111 El Paso & El Paso & $48: 141$ & 479,889 & 12801 & 032 & 03701-03705 \\
\hline \multirow[t]{5}{*}{112 Fort Worth } & Tarrant & $48: 439$ & 860,880 & 11302 & $\begin{array}{l}\text { 019,020, } \\
021\end{array}$ & $\begin{array}{l}\text { 01901-01904, } \\
\text { 02001-02002 } \\
\text { 02101-02104 }\end{array}$ \\
\hline & Johnson & $48: 251$ & 67,649 & 11302 & 025 & 01800 \\
\hline & Hood & $48: 221$ & 17,714 & & 025 & 01800 \\
\hline & Parker & $48: 367$ & 44,609 & & 025 & 01800 \\
\hline & Wise & $48: 497$ & 26,575 & & 025 & 01800 \\
\hline \multicolumn{7}{|c|}{1980 and 1990 match with $7.29 \%$ less than 1970 . } \\
\hline \multirow[t]{7}{*}{113 Houston } & Brazoria & 48:039 & 169,587 & 12502 & 062 & 06400 \\
\hline & Fort Bend & $48: 157$ & 130,846 & 12502 & 063 & $06501-06502$ \\
\hline & Montgomery & $48: 339$ & 128,487 & 12502 & 064 & 06200 \\
\hline & Liberty & 48:291 & 47,088 & 12502 & 064 & \\
\hline & Harris & $48: 201$ & $2,409,547$ & 12502 & 058 - 061 & $\begin{array}{c}06601-06615,06700 \\
06800,06901-06908\end{array}$ \\
\hline & Waller & $48: 473$ & 19,798 & & 063 & \\
\hline & Chambers & 48:071 & 18,538 & & 064 & \\
\hline
\end{tabular}

1970 and 1990 match. 1980 has $1.33 \%$ more.

\begin{tabular}{|c|c|c|c|c|c|c|}
\hline \multirow[t]{5}{*}{114 San Antonio } & Bexar & 48:029 & 988,800 & 12601 & 040,041 & $\begin{array}{c}04401-04408 \\
04501-04502\end{array}$ \\
\hline & Guadalupe & $48: 187$ & 46,708 & 12601 & 042 & 04600 \\
\hline & Comal & 48:091 & 36,446 & & 042 & 04600 \\
\hline & Karnes & $48: 255$ & 13,593 & & 042 & 04600 \\
\hline & Wilson & $48: 493$ & 16,756 & & 042 & 04600 \\
\hline \multicolumn{7}{|c|}{1980 and 1990 match with $4.51 \%$ less than 1970 . } \\
\hline \multicolumn{7}{|l|}{ Utah } \\
\hline \multirow[t]{3}{*}{115 Salt Lake City } & Salt Lake & 49:035 & 619,066 & 13202 & 002,003 & 00400 \\
\hline & Davis & 49:011 & 146,540 & 13202 & 004 & 00300 \\
\hline & Tooele & 49:045 & 26,033 & & & 00300 \\
\hline
\end{tabular}

1970 and 1980 match. 1990 has $3.40 \%$ more. 
Table 4, continued

\begin{tabular}{|c|c|c|c|c|c|c|}
\hline MSA & $\begin{array}{l}1970 \text { Counties/ } \\
\text { Other Counties }\end{array}$ & $\begin{array}{l}\text { Cnty } \\
\text { Num } \\
\end{array}$ & $\begin{array}{l}1980 \\
\text { Pop. }\end{array}$ & $\begin{array}{c}1970 \\
\text { County } \\
\text { Group } \\
\end{array}$ & $\begin{array}{c}1980 \\
\text { County } \\
\text { Group } \\
\end{array}$ & $\begin{array}{c}1990 \\
\text { PUMA } \\
\end{array}$ \\
\hline \multicolumn{7}{|l|}{ Virginia } \\
\hline $\begin{array}{l}116 \text { Newport News } \\
\text { Hampton }\end{array}$ & $\begin{array}{l}\text { York } \\
\text { Hampton } \\
\text { Newport News } \\
\text { Gloucester } \\
\text { James City } \\
\text { Poquoson } \\
\text { Williamsburg } \\
\text { York }\end{array}$ & $\begin{array}{l}51: 199 \\
51: 650 \\
51: 700 \\
51: 073 \\
51: 095 \\
51: 735 \\
51: 830 \\
51: 199\end{array}$ & $\begin{array}{r}35,463 \\
122,617 \\
144,903 \\
20,107 \\
22,763 \\
8,726 \\
9,870 \\
35,463\end{array}$ & $\begin{array}{l}2001 \\
2001 \\
2001\end{array}$ & $\begin{array}{l}\text { 021 } \\
020 \\
021 \\
021 \\
021 \\
021 \\
021\end{array}$ & $\begin{array}{l}\mathbf{0 3 0 0 0} \\
\mathbf{0 3 1 0 0}\end{array}$ \\
\hline \multicolumn{7}{|c|}{ No match. 1980 has $31.99 \%$ more than 1970 and 1990 has $11.70 \%$ less than 1970 . } \\
\hline $\begin{array}{l}117 \text { Norfolk } \\
\text { Portsmouth }\end{array}$ & $\begin{array}{l}\text { Portsmouth } \\
\text { Virginia Beach } \\
\text { Chesapeake } \\
\text { Norfolk } \\
\quad \text { Suffolk }\end{array}$ & $\begin{array}{l}51: 740 \\
51: 810 \\
51: 550 \\
51: 710 \\
51: 800\end{array}$ & $\begin{array}{r}104,577 \\
262,199 \\
114,486 \\
266,979 \\
47,621\end{array}$ & $\begin{array}{l}2003 \\
2003 \\
2003 \\
2002\end{array}$ & $\begin{array}{l}016 \\
019 \\
017 \\
018 \\
016\end{array}$ & $\begin{array}{l}02600 \\
02900 \\
02500 \\
02700\end{array}$ \\
\hline 1970 and 1990 & ch. 1980 has $6.36 \% \mathrm{~m}$ & & & & & \\
\hline 118 Richmond & $\begin{array}{l}\text { Hanover } \\
\text { Henrico } \\
\text { Richmond } \\
\text { Chesterfield } \\
\text { Charles City } \\
\text { Goochland } \\
\text { New Kent } \\
\text { Powhatan }\end{array}$ & $\begin{array}{l}51: 085 \\
51: 087 \\
51: 760 \\
51: 041 \\
51: 036 \\
51: 075 \\
51: 127 \\
51: 145\end{array}$ & $\begin{array}{r}50,398 \\
180,735 \\
219,214 \\
141,372 \\
6,692 \\
11,761 \\
8,781 \\
13,062\end{array}$ & $\begin{array}{l}1901 \\
1901 \\
1901 \\
1901\end{array}$ & $\begin{array}{l}025 \\
025 \\
023 \\
024 \\
025 \\
025 \\
025 \\
025\end{array}$ & $\begin{array}{l}\mathbf{0 2 0 0 0} \\
01900 \\
\mathbf{0 2 1 0 0} \\
\mathbf{0 1 8 0 0} \\
\mathbf{0 2 0 0 0} \\
\mathbf{0 2 0 0 0} \\
\mathbf{0 2 0 0 0} \\
\mathbf{0 2 0 0 0}\end{array}$ \\
\hline
\end{tabular}

1980 and 1990 match with $6.81 \%$ more than 1970 .

\section{Washington}

119 Seattle

Everett

120 Spokane

121 Tacoma

West Virginia

122 Huntington

Asland
King County

Snohomish

Spokane

Pierce

Lawrence, OH
Cabel, WV
Wayne, WV
Boyd, KY
Gallia, OH
Vinton, OH
Jackson,OH
Carter, KY
Elliot,KY
Greenup,KY
Lawrence, KY
Lincoln,WV
Logan,WV
Mason,WV
Mingo, WV

$\begin{array}{lr}53: 033 & 1,269,749 \\ & \\ 53: 061 & 337,720 \\ & \\ 53: 063 & 341,835 \\ & \\ 53: 053 & 485,643 \\ & \\ & \\ 39: 087 & 63,849 \\ 54: 011 & 106,835 \\ 54: 099 & 46,021 \\ 21: 019 & 55,513 \\ 39: 053 & 30,098 \\ 39: 163 & 11,584 \\ 39: 079 & 30,592 \\ 21: 043 & 25,060 \\ 21: 063 & 6,908 \\ 21: 089 & 39,132 \\ 21: 127 & 14,121 \\ 54: 043 & 23,675 \\ 54: 045 & 50,679 \\ 54: 053 & 27,045 \\ 54: 059 & 37,336\end{array}$

13501

13502

1340

13403

005,006

013,014

$\begin{array}{ll}\mathbf{4 8 0 1} & \mathbf{3 9 : 0 5 9} \\ \mathbf{4 8 0 1} & \mathbf{5 4 : 0 1 0} \\ \mathbf{4 8 0 1} & 54: 011 \\ \mathbf{4 8 0 1} & 21: 011 \\ & \mathbf{3 9 : 0 5 9} \\ & \mathbf{3 9 : 0 5 9} \\ & \\ & 21: 011 \\ & 21: 011 \\ & 21: 011 \\ & 21: 011 \\ & 54: 011 \\ & 54: 011 \\ & 54: 011 \\ & 54: 011\end{array}$

01701-01703

01801-01808

01001-01004

00500,00600

01301-01304

No Match. 1980 has $15.31 \%$ more than 1970 in one area and $37.3 \%$ less in another area. 1990 has $73.26 \%$ more than 1970 in one area and $20.39 \%$ less in another. 
Table 4, continued

\begin{tabular}{|c|c|c|c|c|c|c|}
\hline MSA & $\begin{array}{l}1970 \text { Counties/ } \\
\text { Other Counties }\end{array}$ & $\begin{array}{l}\text { Cnty } \\
\text { Num }\end{array}$ & $\begin{array}{l}1980 \\
\text { Pop. }\end{array}$ & $\begin{array}{l}1970 \\
\text { County } \\
\text { Group }\end{array}$ & $\begin{array}{l}1980 \\
\text { County } \\
\text { Group } \\
\end{array}$ & $\begin{array}{c}1990 \\
\text { PUMA } \\
\end{array}$ \\
\hline \multicolumn{7}{|l|}{ Wisconsin } \\
\hline $\begin{array}{l}123 \text { Appleton } \\
\text { Oshkosh }\end{array}$ & $\begin{array}{l}\text { Outagamie } \\
\text { Winnebago } \\
\text { Calumet }\end{array}$ & $\begin{array}{l}55: 087 \\
55: 139 \\
55: 015\end{array}$ & $\begin{array}{r}128,799 \\
131,703 \\
30,867\end{array}$ & $\begin{array}{l}7801 \\
7801 \\
7801\end{array}$ & $\begin{array}{l}005 \\
006 \\
006\end{array}$ & $\begin{array}{l}00400 \\
00500 \\
00400\end{array}$ \\
\hline 124 Madison & Dane & $55: 025$ & 323,545 & 7702 & 017,018 & 01600,01700 \\
\hline 125 Milwaukee & $\begin{array}{l}\text { Milwaukee } \\
\text { Ozaukee } \\
\text { Washington } \\
\text { Waukesha }\end{array}$ & $\begin{array}{l}55: 079 \\
55: 089 \\
55: 131 \\
55: 133\end{array}$ & $\begin{array}{r}964,988 \\
66,981 \\
84,848 \\
280,326\end{array}$ & $\begin{array}{l}7703 \\
7704 \\
7704 \\
7704\end{array}$ & $\begin{array}{c}\text { 023,024 } \\
026 \\
026 \\
025\end{array}$ & $\begin{array}{c}\text { 02201-02206 } \\
02400 \\
02400 \\
02300\end{array}$ \\
\hline
\end{tabular}

NOTES: County group or PUMA numbers in bold are included in the match. Plain text numbers are not. Unless otherwise indicated, the 1970, 1980, and 1990 definitions match exactly. 